\title{
An Example of Sound Insulation Analysis in Building Elements with Different Materials on the Interior Surface
}

\author{
Zuhal Ozcetin \\ Fine Arts and Design Faculty, Department of Architecture, \\ Siirt University, Siirt, Turkey \\ E-mail: zuhalozcetin@gmail.com
}

\begin{abstract}
The aim of this study was to examine and assess the performance of structural elements against applicable standards and to evaluate their implementation and condition with different internal surface materials. Moreover, the compliance status with the "Regulation on the Protection of Buildings against Noise" was evaluated. Building elements in the lecture halls of the education building, were acoustically measured against established standards and the sound transmission loss values of the walls were calculated. The values obtained before the improvement: for the inner wall-1: $\mathrm{R}_{\mathrm{w}}$ value $31(0 ;-1)-\mathrm{D}_{\mathrm{nT}}$ value $30(0 ;-1)$; for the inner wall-2: $\mathrm{R}_{\mathrm{w}}$ value $40(-1 ;-3)-\mathrm{D}_{\mathrm{nT}}$ value $40(-1 ;-3)$. After the improvement: for the inner wall-1: $R_{w}$ value $31(0 ; 0)-D_{n T}$ value $30(0 ;-1)$; for the inner wall-2: $R_{w}$ value $39(-1 ;-3)-D_{n T}$ value 39 $(-1 ;-3)$. These fall below the required limit values. For the inner walls -1 and -2 , it was also determined that the sound transmission loss values were below the limit values before and after the implementation. Solution suggestions include increasing material thickness or using materials with complete laboratory tests.
\end{abstract}

Keywords: Acoustic, silk (live) plaster, building material, building elements.

DOI: $10.7176 /$ JSTR/6-04-11

\section{Introduction}

Sound is the audible waves perceived by the ear. On the other hand, noise can be defined as unwanted sound. Noise is an important environmental problem especially in big cities, it affects hearing health and perception, disrupts physiological and psychological balance, decreases job performance, destroys environmental calm, and reduces quality of life at home, in the workplace, in public transport, and in the street, as it can affect people in their residences, during work, or on the move even as pedestrians (Y 1 maz Demirkale, 2007: 202-465, Kurra, 1997: 31-40).

Human behavior under the influence of noise, which can seriously affect health, can be examined in two aspects: These are psychological and physiological discomfort: the former can be detected through the way feelings and senses are expressed and the latter by various measurement methods. This renders noise as a problem that could and should be solved at the design stage, during the architectural planning of a space. In order to create an auditory-comfortable environment at the design stage, measures should be taken to remove noise from the structure and take it under control. This requirest first to define the highest noise level acceptable in terms of human and public health, then determining the noise conditions by methods of measurement and estimation, and finally ensuring noise control and the homogeneous distribution of noise in the space (Y1lmaz Demirkale, 2007: 202-465, Kurra, 2009: 55-460).

Today, children and young people spend most of their time inside educational buildings. This highlights the crucial importance of designing educational structures with sustainable features. In most of Europe and North America, laws and regulations make it mandatory to provide sustainable features in educational buildings. Certification requirements for educational buildings include receiving adequate daylight, choices of color, energy efficiency in design, better indoor air quality, sharing building facilities with the immediate environment, robustness, use of easy-to-clean materials, and acoustic comfort in classrooms. This, in turn, makes it imperative to plan a sustainable education structure that shows the students how humans can shape and affect the earth and that encourages them to take steps towards a more sustainable living (Bayazıt, 2006: 130-133; Gökçora, 2003: 54).

In addition to planning new educational buildings in line with the regulations, it is important for 
efficiency in education to evaluate the existing ones according to the same guidelines. In the specific example of educational buildings, following complaints of low lecture efficiency in the halls of the Faculty of Economics and Administrative Sciences an investigatation was called for. As a result, a sound insulation assessment was made in the educational facilities before and after the application of the internal surface material for the building elements in terms of auditory comfort as stipulated by the "Regulation on the Protection of Buildings against Noise" and the measurement and calculation methods to obtain the sound transmission loss values were used.

In the specific example of this educational building, a silk (live) plaster material known to provide acoustic effects with heat and sound insulation was used to ensure authenticity. The aim of the study was to analyze the effects of the improvement performed and especially the effect of the building material on the building elements and the compliance of the obtained data with the "Regulation on the Protection of Buildings against Noise".

A lot of misinformation on the subject aside, it is not known if several building materials on the market actually perform as it is stated in their promotional materials. After all, many are claimed to perform very well even though they do not even have their laboratory measurements and analyses done. Lack of informed choices in the purchase of materials and budget limitations are a factor in opting for easy and low-budget solutions in public buildings (and facilities of education mostly fall in this category). Consequently, an assessment of the current situation in terms of acoustic comfort in the lecture halls formed the scope of the study. Furthermore, the silk (live) plaster applied on the existing plastered wall as an improvement and the current condition of the structures were evaluated. In this context, the performance of the building element before and after the implementation was analyzed in order to evaluate it according to the standards of the "Regulation on the Protection of Buildings against Noise."

\section{Method}

The method of this study involved acoustic measurements of the building elements in the lecture halls of the education building, performed against established standards, as well as the calculations of the sound transmission loss values of the walls. Performance analyses in terms of sound loss in the building elements were made before the application (of plaster on brick) and afterwards (plaster on brick + silk [live] plaster).

In the specific example of educational buildings and their building materials, the study focuses on the analysis of the sound transmission loss value of "silk (live) plaster." The sound transmission loss performance of building elements was evaluated by calculating the "Weighted Apparent Sound Reduction Index" $\left(\mathrm{R}_{\mathrm{w}}\right)$ parameter and the "Standardized Level Difference" $\left(\mathrm{D}_{\mathrm{nT}}\right)$ value within the framework of the "Regulation on the Protection of Buildings against Noise."

The problematic of the study was investigating the insufficiency of acoustic comfort conditions in educational buildings (classrooms, lecture halls, etc.) and the performance of the interior surface material used in the walls, be it in a positive or negative way. A sub problematic was the capability of the construction materials in the market to meet the performance limit values of the building elements and unknown interior performance.

Noise problem in educational buildings is sufficiently demonstrated with extensive studies from various countries. In Turkey, the number of comprehensive statistical studies on the subject is limited, yet there is a plethora of academic studies to demonstrate the unacceptable noise levels in educational buildings, from both internal and external sources. Sust and Lazarus state that auditory discomfort creates problems in the communication process at three different levels:

- The content is not fully understood, it is understood incompletely or completely misunderstood.

- Relational and personal statements are misinterpreted.

- Behavioral expectations are not fully perceived, they are perceived incompletely or completely misperceived (Can, 2007: 16).

The level of development of a country or society is directly related to the quality of its education (Öztürk, 2001: 39-47). A good quality education requires comfort conditions for teachers and students (Esin, 2007: 71-74, Petreche et al., 2007: 984-999). Among these conditions of comfort, acoustics constitute an important parameter as it is crucial for the student to hear and understand the teacher and for the teacher to continue the lecture in a comfortable manner. Noise from external environment and poor acoustics of a classroom or lecture hall negatively affect teaching and education for both the teacher and the student 
(Sutherland et al., 2001: 2-7-Federal Interagency Committee on Aviation Noise [FICAN], 2000: 5-40). In buildings with an educational purpose, the materials used affect the sound transmission loss performance of the building elements positively or negatively, besides the whole process of design. A review of studies conducted highlights determining the sound absorption coefficient of porous materials with mathematical models and calculation method, as well as the use of an impedance tube and sound insulation coefficient measurements for the evaluation of the sound insulation capacity of natural fibers. In our study, we investigated how the finishing material used on the inner surface of a building affected the performance of the building element. We also analyzed the reverberation period in the interior space and the change in performance.

\section{Results}

\subsection{Evaluation of the Current Situation}

In this study, the walls of a lecture hall in the Faculty of Economics and Administrative Sciences were analyzed before and after the works of improvement. The measurements were performed when the hall was empty using a sound source of type 122dB-re: $1 \mathrm{pW}$, a power amplifier of 2734-A: (500W), a 4192 $1 \backslash 2$ inch microphone, and a Brüel\&Kjaer microphone preamplifier of the model 2669-B. Measurements required under the standard TS EN ISO 16283-1 for building elements and structures in the classrooms were performed at the designated source and receiver points in accordance with the space/area dimensions, spatial arrangement, and the relation of the space with adjacent spaces. For interior walls, the wall between the lecture hall and the hallway (inner wall-1) and the wall from one lecture hall to the other (inner wall-2) were measured. The material used on the existing walls was known to be a brickbased plaster.

Microphones were calibrated before starting the measurements. This was followed by applying white noise 3 times for 16 seconds each from the source for each receiver point determined according to frequencies and the average sound pressure level was measured in the source and sensing areas (Picture 1-Figure 1).

$\mathrm{K} 2$ sound source was positioned on the same axis as A1 receiver point with $2.5 \mathrm{~m}$ distance to the wall (reflective surface); the receiver points A1, A2, and A3 with $2.5 \mathrm{~m}$ distance to reflective surfaces; K1 sound source on the middle axis with $3.5 \mathrm{~m}$ distance to the reflective surface (inner wall-2); the A4 receiver point with $2.0 \mathrm{~m}$ distance from the reflective surface (inner wall-2) on the center axis; and finally the A5 receiver point with $2.5 \mathrm{~m}$ distance from the side reflective surface and $5 \mathrm{~m}$ from the inner wall-2 (Figure 1).

Receiver and source points were determined as the points of measurement at a short and long distance to the reflective surface, respectively, in accordance with the standards. The points on the same axis were selected. Source and receiver points were designated in the reception and source rooms.

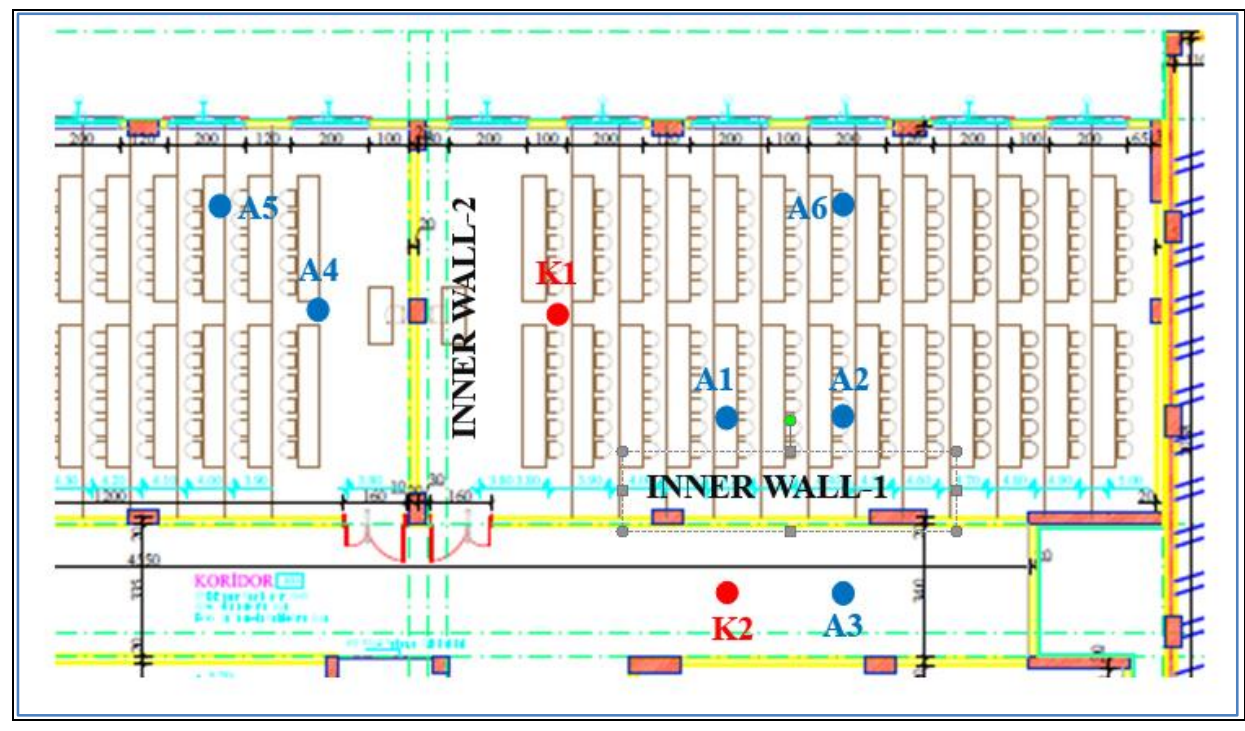

Figure 1. Source and receiver points for the measurements made 
Following the measurements performed in the lecture halls of the Faculty of Economics and Administrative Sciences, the mean values were obtained and the "Weighted Apparent Sound Reduction Index" used to measure the performance of building elements against sound dissipated in the air was calculated with the formula,

$\mathrm{R}_{\mathrm{w}}=\mathrm{L} 1-\mathrm{L} 2+10 \log (\mathrm{S} / \mathrm{A})$.

Where,

$R_{w}$ is the weighted apparent sound reduction index (dB),

L1 is the average sound pressure level $(\mathrm{dB})$ in the source room,

L2 is the average sound pressure level $(\mathrm{dB})$ in the perception room, and

$\mathrm{S}$ is the area of the intermediate wall/floor $\left(\mathrm{m}^{2}\right)$.

A shows the equivalent absorption area $\left(\mathrm{Sabin}, \mathrm{m}^{2}\right)$ in the perception room.

Equivalent absorption area is calculated with the following formula:

$\mathrm{A}=\frac{0,16 \mathrm{~V}}{T}$

Where,

A: equivalent sound absorption area in the perception room based on frequency (sound absorbing surface area), expressed as $\mathrm{m}^{2}$

$\mathrm{V}$ : volume of the perception room as $\mathrm{m}^{3}$

$\mathrm{T}$ : time of reverberation in the perception room as seconds.
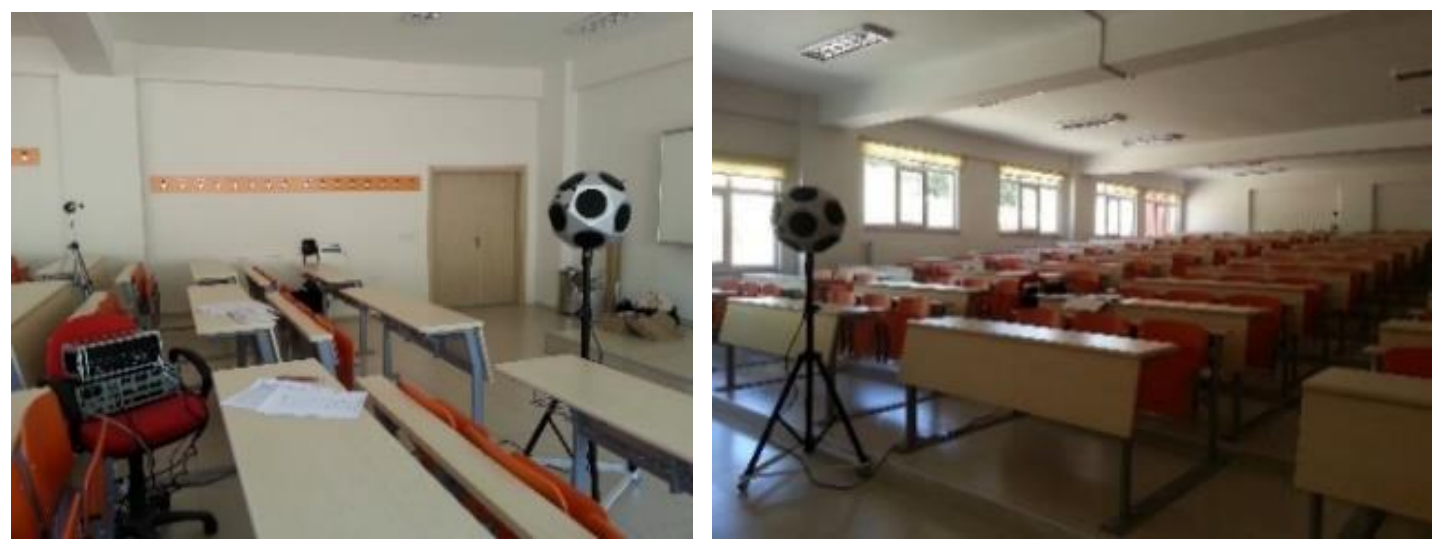

Picture 1. Photos before application

\subsection{Evaluation of the Pre-Improvement Acoustic Comfort in the Lecture Halls}

In the lecture hall of an area of approximately $190 \mathrm{~m}^{2}$, the building material used is plaster on brick. Table 1 shows the pre-improvement sound pressure level measurement values for inner wall-1 based on frequency.

Table 1. Sound pressure level measurement values for inner wall - 1 (before application)

\begin{tabular}{|c|c|c|c|c|c|c|c|c|c|c|}
\hline & $100 \mathrm{~Hz}$ & $\begin{array}{l}125 \\
\mathrm{~Hz}\end{array}$ & $160 \mathrm{~Hz}$ & $200 \mathrm{~Hz}$ & $250 \mathrm{~Hz}$ & $315 \mathrm{~Hz}$ & $400 \mathrm{~Hz}$ & $500 \mathrm{~Hz}$ & $630 \mathrm{~Hz}$ & $800 \mathrm{~Hz}$ \\
\hline$K_{2}-A_{1}$ & 73,90 & 71,40 & 76,00 & 72,10 & 69,70 & 66,70 & 66,20 & 63,20 & 61,10 & 60,60 \\
\hline $\mathbf{K}_{2}-\mathbf{A}_{2}$ & 69,80 & 71,00 & 72,10 & 69,70 & 68,80 & 65,00 & 64,10 & 61,40 & 59,90 & 58,30 \\
\hline \multirow[t]{3}{*}{$\mathbf{K}_{2}-\mathbf{A}_{3}$} & 99,30 & 96,00 & 96,70 & 96,90 & 96,80 & 94,40 & 94,90 & 91,80 & 91,90 & 90,10 \\
\hline & 1000 & 1250 & 1600 & 2000 & 2500 & 3150 & 4000 & $5000 \mathrm{~Hz}$ & $6300 \mathrm{~Hz}$ & $8000 \mathrm{~Hz}$ \\
\hline & $\mathrm{Hz}$ & $\mathrm{Hz}$ & $\mathrm{Hz}$ & $\mathrm{Hz}$ & $\mathrm{Hz}$ & $\mathrm{Hz}$ & $\mathrm{Hz}$ & & & \\
\hline $\mathbf{K}_{2}-\mathbf{A}_{1}$ & 58,60 & 60,50 & 61,60 & 60,60 & 61,50 & 59,20 & 54,90 & 55,40 & 49,90 & 41,00 \\
\hline $\mathbf{K}_{2}-\mathbf{A}_{2}$ & 58,20 & 60,00 & 60,10 & 58,10 & 59,70 & 57,90 & 52,70 & 53,10 & 47,60 & 38,20 \\
\hline $\mathbf{K}_{2}-\mathbf{A}_{3}$ & 89,00 & 89,80 & 90,40 & 89,40 & 90,50 & 89,60 & 87,40 & 90,30 & 86,70 & 78,60 \\
\hline
\end{tabular}

Table 2 shows the results of measurement and calculation of the sound transmission loss values for inner wall-1. 
International Journal of Scientific and Technological Research

Table 2. Results of the pre-application measurement and calculation for the sound transmission loss value of the inner wall- 1

\begin{tabular}{|c|c|c|c|c|c|}
\hline \multirow[t]{2}{*}{ Frequency $(\mathrm{Hz})$} & \multirow[t]{2}{*}{$\mathbf{T}_{30}$} & \multirow{2}{*}{$\begin{array}{c}\mathrm{L}_{1}(\mathrm{~dB}) \\
\mathrm{A} 3\end{array}$} & \multicolumn{2}{|c|}{$L_{2}(d B)$} & \multirow[t]{2}{*}{$R_{w}=L_{1}-L_{2}+10 \log (S / A)(d B)$} \\
\hline & & & A1 & A2 & \\
\hline 100 & 3,25 & 99,3 & 73,90 & 69,80 & 36,0 \\
\hline 125 & 3,99 & 96,0 & 71,40 & 71,00 & 34,3 \\
\hline 160 & 3,64 & 96,7 & 76,00 & 72,10 & 31,7 \\
\hline 200 & 3,80 & 96,9 & 72,10 & 69,70 & 35,3 \\
\hline 250 & 3,32 & 96,8 & 69,70 & 68,80 & 36,2 \\
\hline 315 & 2,27 & 94,4 & 66,70 & 65,00 & 35,5 \\
\hline 400 & 2,41 & 94,9 & 66,20 & 64,10 & 37,0 \\
\hline 500 & 2,32 & 91,8 & 63,20 & 61,40 & 36,6 \\
\hline 630 & 2,06 & 91,9 & 61,10 & 59,90 & 38,0 \\
\hline 800 & 2,23 & 90,1 & 60,60 & 58,30 & 37,6 \\
\hline 1000 & 2,30 & 89,0 & 58,60 & 58,20 & 37,7 \\
\hline 1250 & 2,48 & 89,8 & 60,50 & 60,00 & 36,9 \\
\hline 1600 & 2,55 & 90,4 & 61,60 & 60,10 & 37,1 \\
\hline 2000 & 2,44 & 89,4 & 60,60 & 58,10 & 37,4 \\
\hline 2500 & 2,26 & 90,5 & 61,50 & 59,70 & 36,9 \\
\hline 3150 & 2,07 & 89,6 & 59,20 & 57,90 & 37,6 \\
\hline 4000 & 1,88 & 87,4 & 54,90 & 52,70 & 39,8 \\
\hline 5000 & 1,65 & 90,3 & 55,40 & 53,10 & 41,7 \\
\hline
\end{tabular}

Figure 2 shows the evaluation of the sound transmission loss value for the inner wall-1 based on the reference curves in the standard TS EN ISO 717-1.

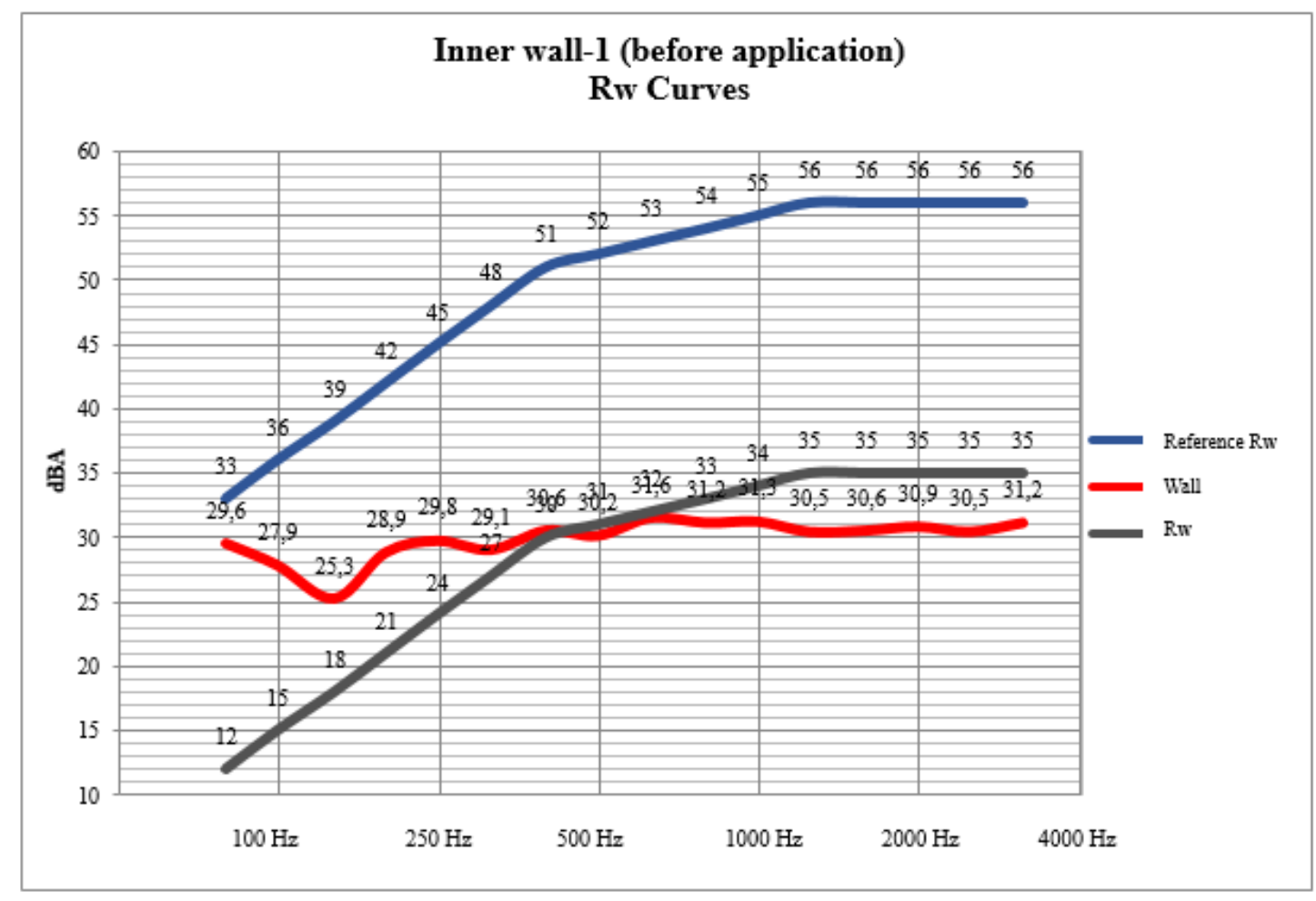

Figure 2. Inner Wall-1 (before application) $\mathrm{R}_{\mathrm{w}}$ graphic

Table 3 shows the pre-improvement measurement values for the sound pressure level of inner wall-1 based on frequency. 
Table 3. Sound pressure level measurement values for inner wall - 2 (before application)

\begin{tabular}{|c|c|c|c|c|c|c|c|c|c|c|}
\hline & $\mathbf{1 0 0} \mathbf{~ H z}$ & $\mathbf{1 2 5} \mathbf{~ H z}$ & $\mathbf{1 6 0} \mathbf{~ H z}$ & $\mathbf{2 0 0} \mathbf{~ H z}$ & $\mathbf{2 5 0} \mathbf{~ H z}$ & $\mathbf{3 1 5} \mathbf{~ H z}$ & $\mathbf{4 0 0} \mathbf{~ H z}$ & $\mathbf{5 0 0} \mathbf{~ H z}$ & $\mathbf{6 3 0} \mathbf{~ H z}$ & $\mathbf{8 0 0} \mathbf{~ H z}$ \\
\hline $\mathbf{K}_{\mathbf{1}}-\mathbf{A}_{\mathbf{1}}$ & 93,80 & 97,70 & 95,10 & 95,20 & 94,30 & 91,10 & 90,70 & 88,70 & 88,40 & 87,10 \\
\hline $\mathbf{K}_{\mathbf{1}}-\mathbf{A}_{\mathbf{6}}$ & 91,50 & 97,30 & 96,40 & 94,30 & 93,00 & 90,90 & 89,90 & 87,80 & 87,20 & 85,80 \\
\hline $\mathbf{K}_{\mathbf{1}}-\mathbf{A}_{\mathbf{4}}$ & 64,50 & 68,70 & 66,40 & 67,60 & 65,90 & 61,60 & 55,80 & 51,70 & 46,70 & 44,70 \\
\hline $\mathbf{K}_{\mathbf{1}}-\mathbf{A}_{\mathbf{5}}$ & 59,90 & 66,70 & 66,10 & 66,20 & 63,10 & 60,10 & 55,80 & 51,10 & 46,60 & 44,40 \\
\hline & $\mathbf{1 0 0 0}$ & $\mathbf{1 2 5 0}$ & $\mathbf{1 6 0 0}$ & $\mathbf{2 0 0 0}$ & $\mathbf{2 5 0 0} \mathbf{~ H z}$ & $\mathbf{3 1 5 0}$ & $\mathbf{4 0 0 0}$ & $\mathbf{5 0 0 0}$ & $\mathbf{6 3 0 0}$ & $\mathbf{8 0 0 0} \mathbf{~ H z}$ \\
\hline & $\mathbf{H z}$ & $\mathbf{H z}$ & $\mathbf{H z}$ & $\mathbf{H z}$ & & $\mathbf{H z}$ & $\mathbf{H z}$ & $\mathbf{H z}$ & $\mathbf{H z}$ & \\
\hline $\mathbf{K}_{\mathbf{1}}-\mathbf{A}_{\mathbf{1}}$ & 85,20 & 89,10 & 87,90 & 86,40 & 87,90 & 87,80 & 84,60 & 86,70 & 82,80 & 74,40 \\
\hline $\mathbf{K}_{\mathbf{1}}-\mathbf{A}_{\mathbf{6}}$ & 84,40 & 87,20 & 87,50 & 85,50 & 86,50 & 86,10 & 83,30 & 85,00 & 81,80 & 73,20 \\
\hline $\mathbf{K}_{\mathbf{1}}-\mathbf{A}_{\mathbf{4}}$ & 44,50 & 43,40 & 45,10 & 44,30 & 45,00 & 42,60 & 36,00 & 36,30 & 31,90 & - \\
\hline $\mathbf{K}_{\mathbf{1}}-\mathbf{A}_{\mathbf{5}}$ & 44,50 & 43,30 & 44,40 & 44,10 & 44,40 & 42,30 & 35,30 & 36,00 & 31,40 & - \\
\hline
\end{tabular}

Measurement and calculation results of the sound transmission loss value for inner wall-2 are in Table 4.

Table 4. Pre-improvement measurement and calculation results of the sound transmission loss value for inner wall-2

\begin{tabular}{|c|c|c|c|c|c|c|}
\hline \multirow{2}{*}{$\begin{array}{l}\text { Frequency } \\
\text { (Hz) }\end{array}$} & \multirow[t]{2}{*}{$\mathbf{T}_{30}$} & \multicolumn{2}{|c|}{$\mathrm{L}_{1}(\mathrm{~dB})$} & \multicolumn{2}{|c|}{$\mathrm{L}_{2}(\mathrm{~dB})$} & \multirow[t]{2}{*}{$\mathbf{R}_{\mathrm{w}}=\mathrm{L}_{1}-\mathrm{L}_{2}+10 \log (\mathrm{S} / \mathrm{A})(\mathrm{dB})$} \\
\hline & & A1 & A6 & A4 & A5 & \\
\hline 100 & 3,38 & 93,80 & 91,50 & 64,50 & 59,90 & 31,6 \\
\hline 125 & 3,86 & 97,70 & 97,30 & 68,70 & 66,70 & 31,8 \\
\hline 160 & 3,76 & 95,10 & 96,40 & 66,40 & 66,10 & 31,1 \\
\hline 200 & 3,67 & 95,20 & 94,30 & 67,60 & 66,20 & 29,7 \\
\hline 250 & 2,95 & 94,30 & 93,00 & 65,90 & 63,10 & 30,4 \\
\hline 315 & 1,99 & 91,10 & 90,90 & 61,60 & 60,10 & 29,7 \\
\hline 400 & 2,02 & 90,70 & 89,90 & 55,80 & 55,80 & 34,4 \\
\hline 500 & 2,12 & 88,70 & 87,80 & 51,70 & 51,10 & 36,5 \\
\hline 630 & 1,73 & 88,40 & 87,20 & 46,70 & 46,60 & 40,3 \\
\hline 800 & 1,68 & 87,10 & 85,80 & 44,70 & 44,40 & 41,4 \\
\hline 1000 & 1,71 & 85,20 & 84,40 & 44,50 & 44,50 & 40,0 \\
\hline 1250 & 1,67 & 89,10 & 87,20 & 43,40 & 43,30 & 44,8 \\
\hline 1600 & 1,51 & 87,90 & 87,50 & 45,10 & 44,40 & 43,1 \\
\hline 2000 & 1,40 & 86,40 & 85,50 & 44,30 & 44,10 & 41,7 \\
\hline 2500 & 1,20 & 87,90 & 86,50 & 45,00 & 44,40 & 42,1 \\
\hline 3150 & 0,99 & 87,80 & 86,10 & 42,60 & 42,30 & 43,7 \\
\hline 4000 & 0,86 & 84,60 & 83,30 & 36,00 & 35,30 & 47,1 \\
\hline 5000 & 0,73 & 86,70 & 85,00 & 36,30 & 36,00 & 47,9 \\
\hline
\end{tabular}

Figure 3 shows the evaluation of the sound transmission loss value for the inner wall-2 based on the reference curves in the standard TS EN ISO 717-1. 


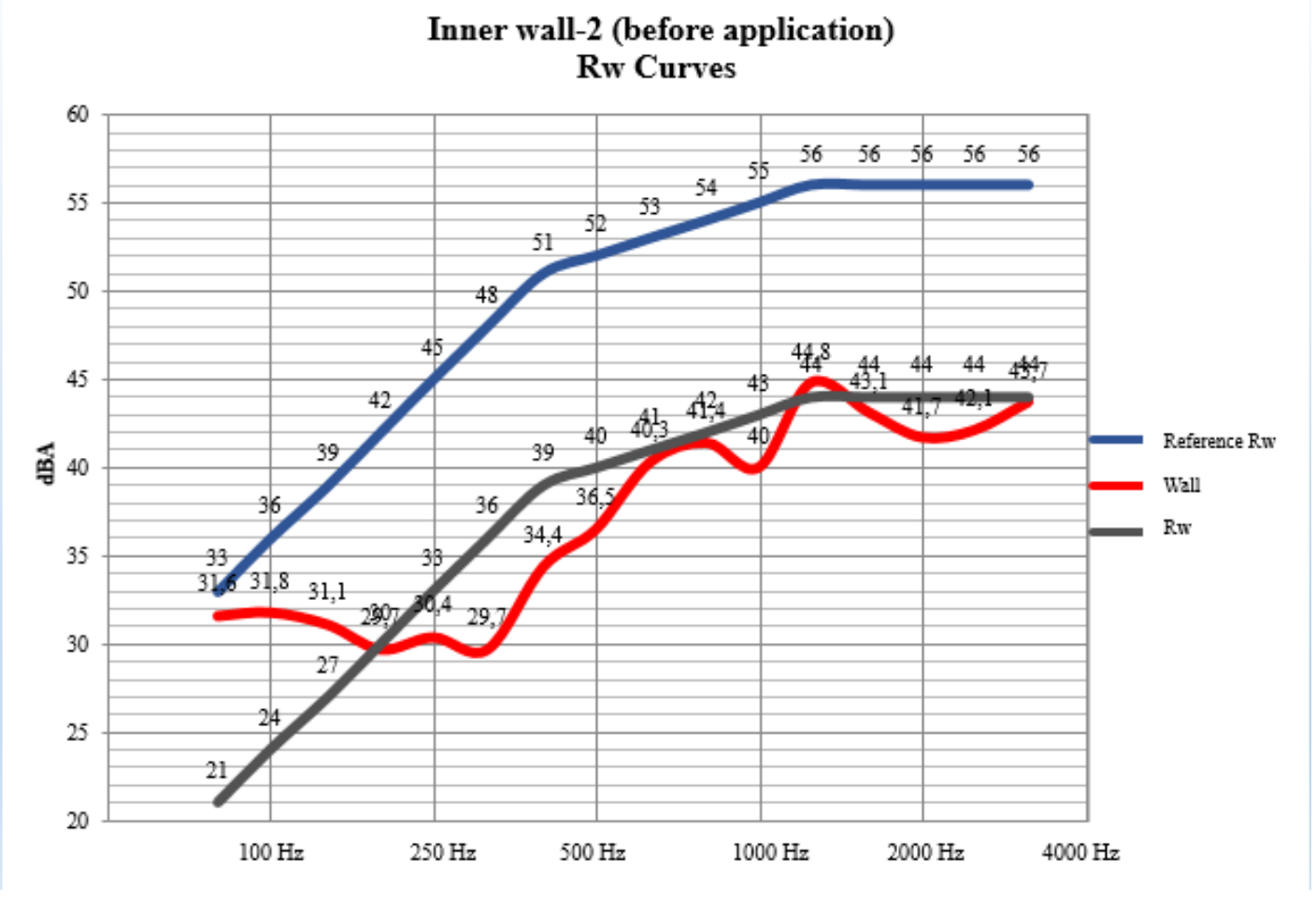

Figure 3. Inner wall-2 (before application) $\mathrm{R}_{\mathrm{w}}$ graphic

\subsection{Evaluation of the Post-Improvement Acoustic Comfort of the Building Elements}

The walls were made of brick with plaster coating before the improvement, which involved applying a coat of silk (live) plaster on the existing coating. This material is in the form of cotton approximately 2 $3 \mathrm{~mm}$ thick. Silk (living) plaster is a building material made of natural fiber, Styrofoam, cellulose, and silk. It is known to provide acoustic effects besides heat and sound insulation. Not only is its application easier compared to paint or wallpaper, but silk (live) plaster lasts longer, as well.
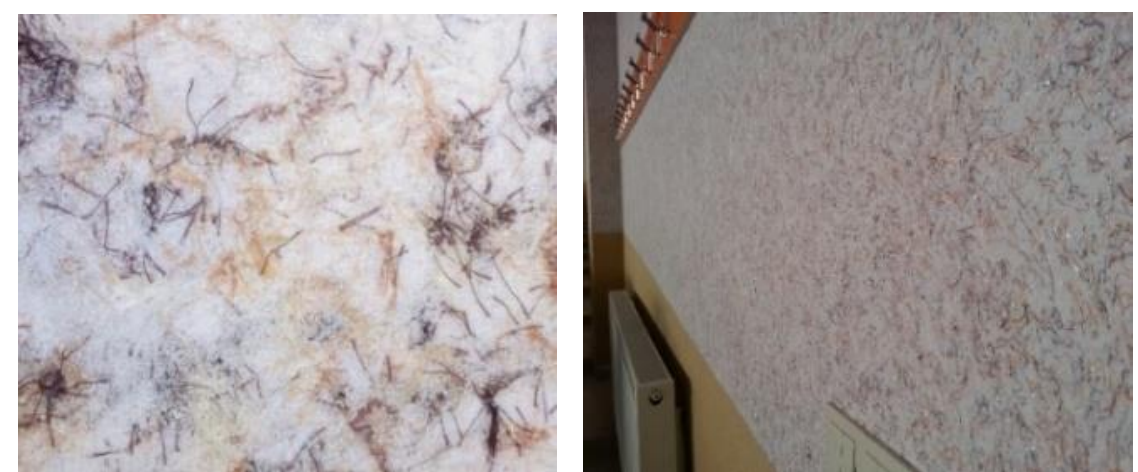

Picture 2. Silk (live) plaster detail made during the application phase 

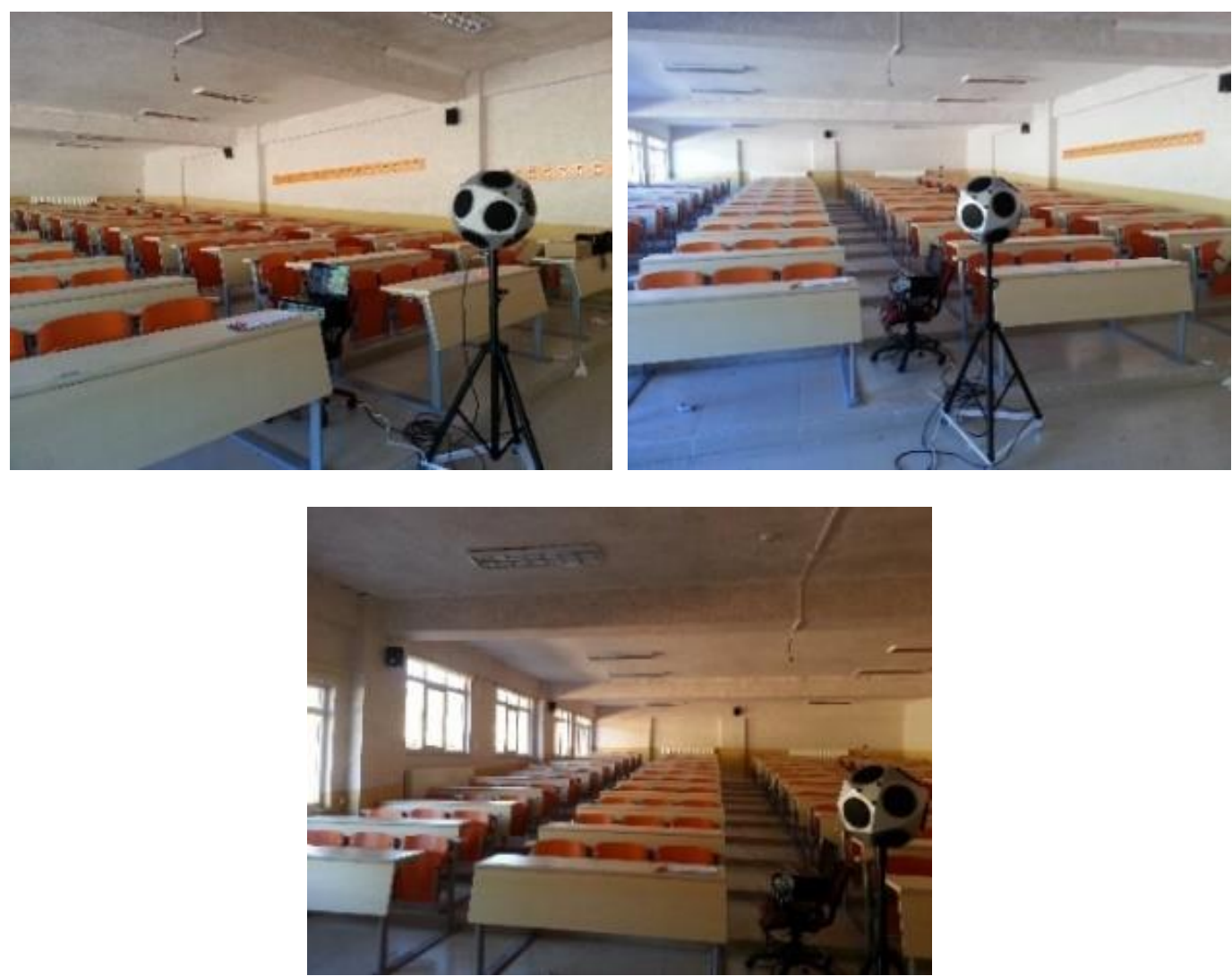

Picture 3. Photos from measurements made after application

The post-improvement values of the sound pressure level measurement for the inner wall-1 are given in Table 5 based on frequency.

Table 5. Sound pressure level measurement values for inner wall - 1 (after application)

\begin{tabular}{|l|c|c|c|c|c|c|c|c|c|c|}
\hline & $\mathbf{1 0 0} \mathbf{~ H z}$ & $\mathbf{1 2 5} \mathbf{~ H z}$ & $\mathbf{1 6 0} \mathbf{~ H z}$ & $\mathbf{2 0 0} \mathbf{~ H z}$ & $\mathbf{2 5 0} \mathbf{~ H z}$ & $\mathbf{3 1 5} \mathbf{~ H z}$ & $\mathbf{4 0 0} \mathbf{~ H z}$ & $\mathbf{5 0 0} \mathbf{~ H z}$ & $\mathbf{6 3 0} \mathbf{~ H z}$ & $\mathbf{8 0 0} \mathbf{~ H z}$ \\
\hline K2-A1 & 78,90 & 77,80 & 80,90 & 80,90 & 75,10 & 74,10 & 72,70 & 70,90 & 66,50 & 67,00 \\
\hline K2-A2 & 72,50 & 80,00 & 82,10 & 79,70 & 74,40 & 71,50 & 69,80 & 69,00 & 66,50 & 65,50 \\
\hline K2-A3 & 105,50 & 104,00 & 106,90 & 103,90 & 106,50 & 102,80 & 100,10 & 99,00 & 99,40 & 96,60 \\
\hline
\end{tabular}

\begin{tabular}{|l|c|c|c|c|c|c|c|c|c|c|}
\hline & $\begin{array}{c}\mathbf{1 0 0 0} \\
\mathbf{H z}\end{array}$ & $\begin{array}{c}\mathbf{1 2 5 0} \\
\mathbf{H z}\end{array}$ & $\begin{array}{c}\mathbf{1 6 0 0} \\
\mathbf{H z}\end{array}$ & $\begin{array}{c}\mathbf{2 0 0 0} \\
\mathbf{H z}\end{array}$ & $\begin{array}{c}\mathbf{2 5 0 0} \\
\mathbf{H z}\end{array}$ & $\begin{array}{c}\mathbf{3 1 5 0} \\
\mathbf{H z}\end{array}$ & $\begin{array}{c}\mathbf{4 0 0 0} \\
\mathbf{H z}\end{array}$ & $\begin{array}{c}\mathbf{5 0 0 0} \\
\mathbf{H z}\end{array}$ & $\begin{array}{c}\mathbf{6 3 0 0} \\
\mathbf{H z}\end{array}$ & $\begin{array}{c}\mathbf{8 0 0 0} \\
\mathbf{H z}\end{array}$ \\
\hline K2-A1 & 65,10 & 66,50 & 67,40 & 65,10 & 66,70 & 62,70 & 58,80 & 57,90 & 54,10 & 42,70 \\
\hline K2-A2 & 63,40 & 65,00 & 65,20 & 63,50 & 63,80 & 61,00 & 55,90 & 56,00 & 51,00 & 40,00 \\
\hline K2-A3 & 97,30 & 98,20 & 98,80 & 97,20 & 98,30 & 96,60 & 94,60 & 97,00 & 93,60 & 85,20 \\
\hline
\end{tabular}

Table 6 shows the measurement and calculation results of sound transmission loss value for the inner wall-1. 
Table 6. Post-improvement measurement and calculation results of sound transmission loss value for the inner wall-1

\begin{tabular}{|c|c|c|c|c|c|}
\hline \multirow{2}{*}{$\begin{array}{l}\text { Frequency } \\
\text { (Hz) }\end{array}$} & \multirow[t]{2}{*}{$\mathbf{T}_{30}$} & \multirow{2}{*}{$\begin{array}{c}\mathrm{L}_{1}(\mathrm{~dB}) \\
\mathrm{A} 3\end{array}$} & \multicolumn{2}{|c|}{$\mathrm{L}_{2}(\mathrm{~dB})$} & \multirow[t]{2}{*}{$R_{w}=L_{1}-L_{2}+10 \log (S / A)(d B)$} \\
\hline & & & A1 & A2 & \\
\hline 100 & 3,25 & 105,50 & 78,90 & 72,50 & 32,2 \\
\hline 125 & 3,99 & 104,00 & 77,80 & 80,00 & 28,0 \\
\hline 160 & 3,64 & 106,90 & 80,90 & 82,10 & 28,2 \\
\hline 200 & 3,80 & 103,90 & 80,90 & 79,70 & 26,3 \\
\hline 250 & 3,32 & 106,50 & 75,10 & 74,40 & 33,5 \\
\hline 315 & 2,27 & 102,80 & 74,10 & 71,50 & 30,1 \\
\hline 400 & 2,41 & 100,10 & 72,70 & 69,80 & 29,0 \\
\hline 500 & 2,32 & 99,00 & 70,90 & 69,00 & 29,4 \\
\hline 630 & 2,06 & 99,40 & 66,50 & 66,50 & 32,4 \\
\hline 800 & 2,23 & 96,60 & 67,00 & 65,50 & 29,7 \\
\hline 1000 & 2,30 & 97,30 & 65,10 & 63,40 & 32,5 \\
\hline 1250 & 2,48 & 98,20 & 66,50 & 65,00 & 31,8 \\
\hline 1600 & 2,55 & 98,80 & 67,40 & 65,20 & 31,4 \\
\hline 2000 & 2,44 & 97,20 & 65,10 & 63,50 & 31,4 \\
\hline 2500 & 2,26 & 98,30 & 66,70 & 63,80 & 30,9 \\
\hline 3150 & 2,07 & 96,60 & 62,70 & 61,00 & 31,8 \\
\hline 4000 & 1,88 & 94,60 & 58,80 & 55,90 & 33,7 \\
\hline 5000 & 1,65 & 97,00 & 57,90 & 56,00 & 35,8 \\
\hline
\end{tabular}

Figure 4 shows the evaluation of the sound transmission loss value for the inner wall-1 based on the reference curves in the standard TS EN ISO 717-1.

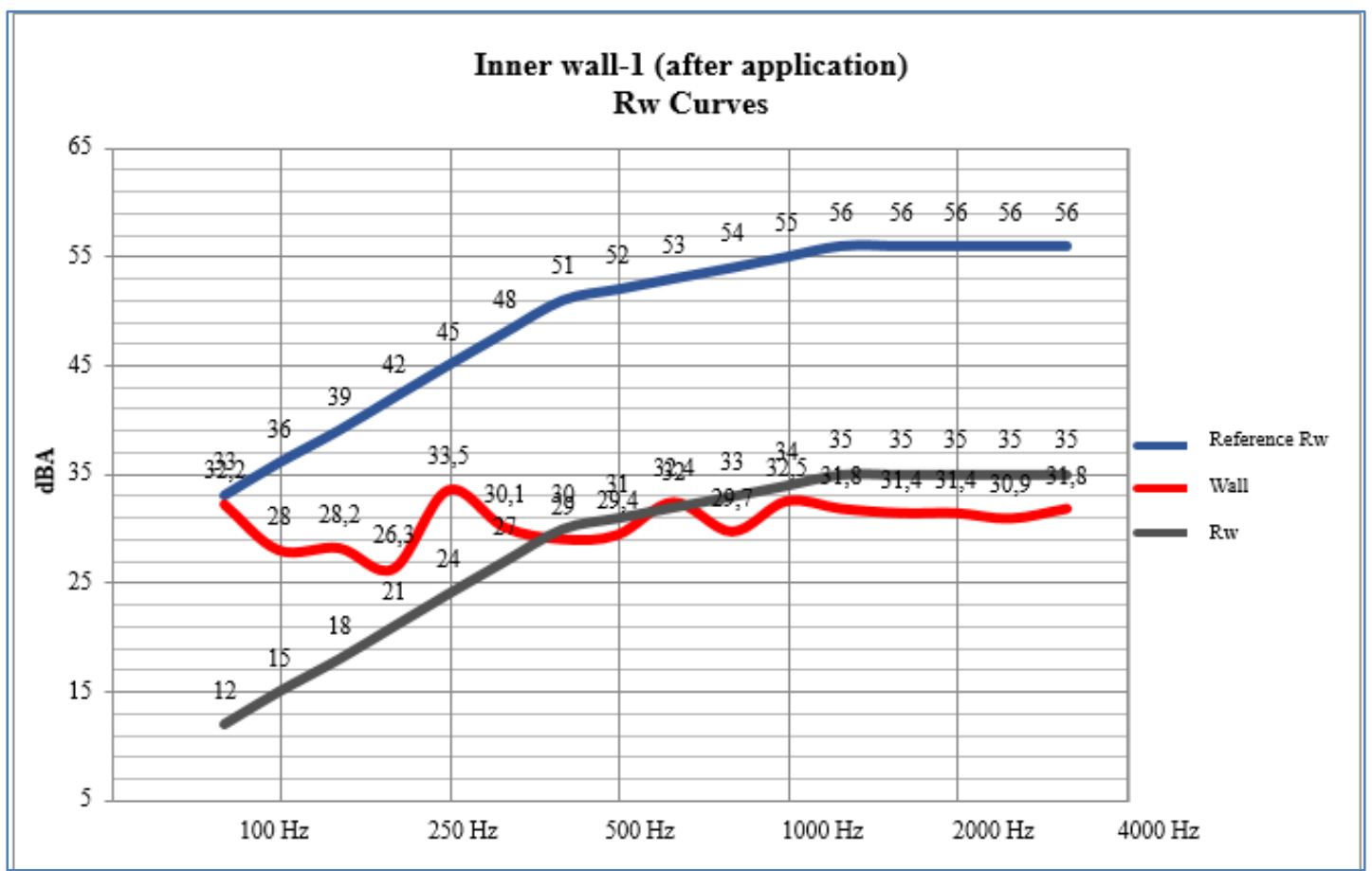

Figure 4. Inner wall-1 (after application) Rw graphic

The post-improvement values of the sound pressure level measurement for the inner wall-2 are given in Table 7 based on frequency. 
International Journal of Scientific and Technological Research

ISSN 2422-8702 (Online), DOI: 10.7176/JSTR/6-04-11

Vol.6, No.4, 2020

Table 7. Post-improvement sound pressure level measurement values for inner wall-2

\begin{tabular}{|c|c|c|c|c|c|c|c|c|c|c|}
\hline & $100 \mathrm{~Hz}$ & $125 \mathrm{~Hz}$ & $160 \mathrm{~Hz}$ & $200 \mathrm{~Hz}$ & $250 \mathrm{~Hz}$ & $315 \mathrm{~Hz}$ & $400 \mathrm{~Hz}$ & $500 \mathrm{~Hz}$ & $630 \mathrm{~Hz}$ & $800 \mathrm{~Hz}$ \\
\hline$K_{1}-A_{1}$ & 98,90 & 106,30 & 102,30 & 102,80 & 100,10 & 98,10 & 98,40 & 96,60 & 96,50 & 93,50 \\
\hline$K_{1}-A_{6}$ & 95,20 & 101,00 & 103,10 & 104,80 & 100,10 & 97,60 & 98,00 & 95,80 & 93,70 & 93,50 \\
\hline $\mathbf{K}_{1}-\mathbf{A}_{4}$ & 74,70 & 73,70 & 74,40 & 75,40 & 72,30 & 69,50 & 64,70 & 59,10 & 54,40 & 53,00 \\
\hline \multirow[t]{2}{*}{$\mathbf{K}_{1}-\mathbf{A}_{5}$} & 70,20 & 76,10 & 76,30 & 75,20 & 70,90 & 67,00 & 63,00 & 58,20 & 53,40 & 51,30 \\
\hline & $1000 \mathrm{~Hz}$ & $1250 \mathrm{~Hz}$ & $\begin{array}{c}1600 \\
\mathrm{~Hz}\end{array}$ & $\begin{array}{c}2000 \\
\mathrm{~Hz}\end{array}$ & $2500 \mathrm{~Hz}$ & $\begin{array}{c}3150 \\
\mathrm{~Hz}\end{array}$ & $\begin{array}{c}4000 \\
\mathrm{~Hz}\end{array}$ & $\begin{array}{c}5000 \\
\mathrm{~Hz}\end{array}$ & $\begin{array}{c}6300 \\
\mathrm{~Hz}\end{array}$ & $8000 \mathrm{~Hz}$ \\
\hline $\mathbf{K}_{1}-\mathbf{A}_{1}$ & 94,20 & 93,30 & 95,90 & 92,80 & 93,00 & 91,50 & 89,10 & 91,30 & 88,10 & 80,70 \\
\hline $\mathbf{K}_{1}-\mathbf{A}_{6}$ & 92,60 & 94,10 & 93,40 & 90,60 & 91,70 & 89,90 & 86,90 & 88,30 & 86,30 & 77,00 \\
\hline $\mathbf{K}_{1}-\mathbf{A}_{4}$ & 51,00 & 49,80 & 51,00 & 49,90 & 49,90 & 46,00 & 39,00 & 37,60 & 32,60 & - \\
\hline$K_{1}-A_{5}$ & 50,20 & 49,80 & 49,20 & 49,00 & 49,10 & 45,80 & 38,70 & 37,60 & 32,10 & - \\
\hline
\end{tabular}

Measurement and calculation results of the sound transmission loss value for inner wall-2 are in Table 8 .

Table 8. Post-improvement measurement and calculation results of sound transmission loss value for the inner wall-2

\begin{tabular}{|c|c|c|c|c|c|c|}
\hline \multirow{2}{*}{$\begin{array}{l}\text { Frequency } \\
(\mathrm{Hz})\end{array}$} & \multirow[t]{2}{*}{$\mathbf{T}_{30}$} & \multicolumn{2}{|c|}{$L_{1}(d B)$} & \multicolumn{2}{|c|}{$\mathrm{L}_{2}(\mathrm{~dB})$} & \multirow{2}{*}{$\underset{(\mathrm{d}}{\mathbf{R}_{\mathrm{w}}=\mathbf{L}_{1}-\mathbf{L}_{2}+10 \log (\mathrm{S} / \mathrm{A})}$} \\
\hline & & A1 & A6 & A4 & A5 & \\
\hline 100 & 3,38 & 98,90 & 95,20 & 74,70 & 70,20 & 25,9 \\
\hline 125 & 3,86 & 106,30 & 101,00 & 73,70 & 76,10 & 30,7 \\
\hline 160 & 3,76 & 102,30 & 103,10 & 74,40 & 76,30 & 29,1 \\
\hline 200 & 3,67 & 102,80 & 104,80 & 75,40 & 75,20 & 30,2 \\
\hline 250 & 2,95 & 100,10 & 100,10 & 72,30 & 70,90 & 29,2 \\
\hline 315 & 1,99 & 98,10 & 97,60 & 69,50 & 67,00 & 28,6 \\
\hline 400 & 2,02 & 98,40 & 98,00 & 64,70 & 63,00 & 33,4 \\
\hline 500 & 2,12 & 96,60 & 95,80 & 59,10 & 58,20 & 36,8 \\
\hline 630 & 1,73 & 96,50 & 93,70 & 54,40 & 53,40 & 39,6 \\
\hline 800 & 1,68 & 93,50 & 93,50 & 53,00 & 51,30 & 39,6 \\
\hline 1000 & 1,71 & 94,20 & 92,60 & 51,00 & 50,20 & 41,2 \\
\hline 1250 & 1,67 & 93,30 & 94,10 & 49,80 & 49,80 & 42,2 \\
\hline 1600 & 1,51 & 95,90 & 93,40 & 51,00 & 49,20 & 42,4 \\
\hline 2000 & 1,40 & 92,80 & 90,60 & 49,90 & 49,00 & 39,7 \\
\hline 2500 & 1,20 & 93,00 & 91,70 & 49,90 & 49,10 & 39,7 \\
\hline 3150 & 0,99 & 91,50 & 89,90 & 46,00 & 45,80 & 40,8 \\
\hline 4000 & 0,86 & 89,10 & 86,90 & 39,00 & 38,70 & 44,5 \\
\hline 5000 & 0,73 & 91,30 & 88,30 & 37,60 & 37,60 & 46,9 \\
\hline
\end{tabular}

Figure 5 shows the evaluation of the sound transmission loss value for the inner wall-2 based on the reference curves in the standard TS EN ISO 717-1. 
International Journal of Scientific and Technological Research

ISSN 2422-8702 (Online), DOI: 10.7176/JSTR/6-04-11

IISE

Vol.6, No.4, 2020

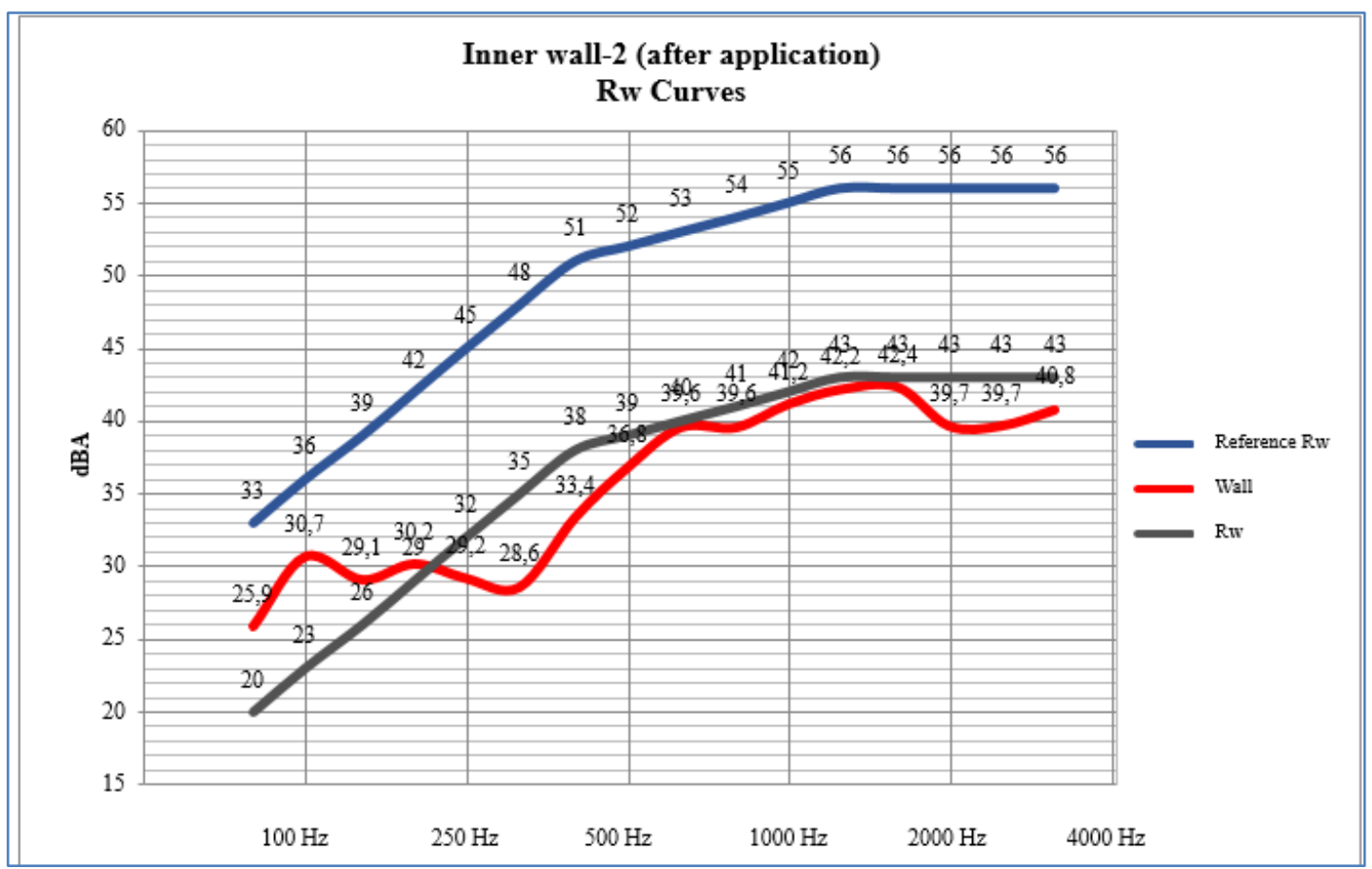

Figure 5. Inner wall-2 (after application) Rw graphic

Figure 6 shows the evaluation of the sound transmission loss value for the inner wall-1 based on the reference curves in the standard TS EN ISO 717-1.

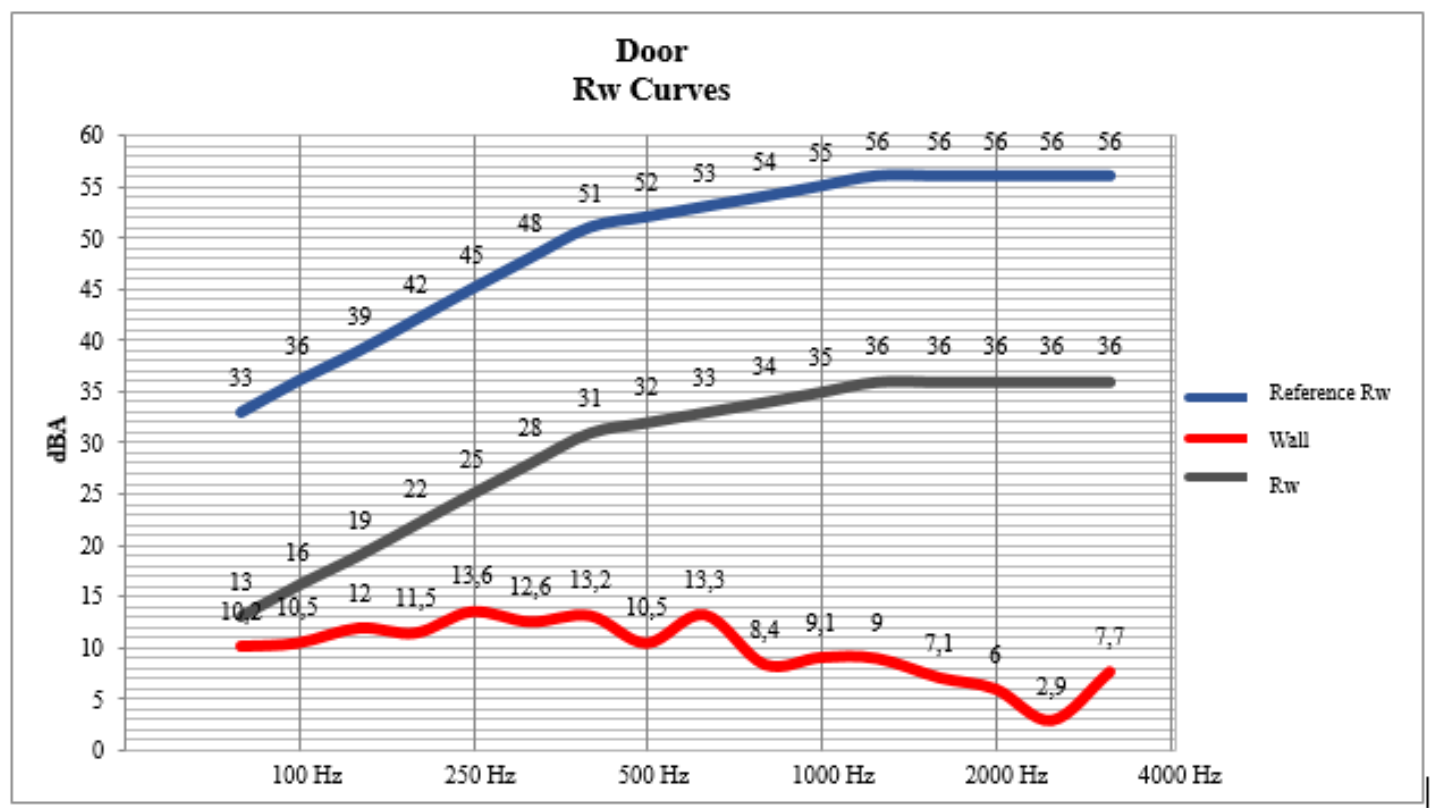

Figure 6. Rw graphic of the door 


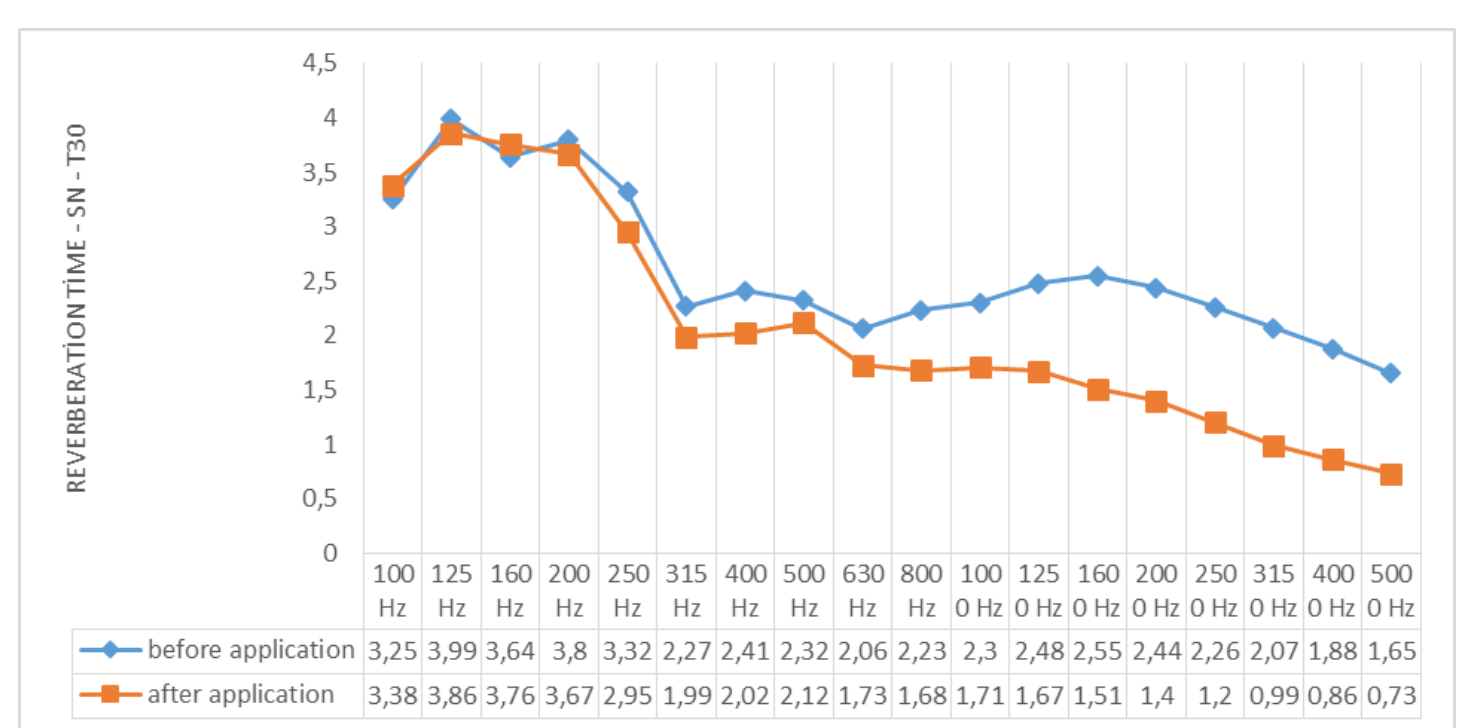

Figure 7. Reverberation time values before and after application

There was a significant decrease in the reverberation time at frequencies as high as $630 \mathrm{~Hz}$ after the application of silk (live) plaster. This indicates for the reverberation time at the lecture halls, silk (live) plaster shows a better performance at higher frequencies.

\section{Conclusion}

In the lecture halls of the Faculty of Economics and Administrative Sciences, the inner walls 1 and 2 were analyzed before and after the improvement with the application of silk (live) plaster on the existing wall coating using the on-site measurement method and the performance of the building elements was evaluated against the "Regulation on Noise Protection of Buildings" in effect in Turkey. According to this, the values below were obtained:

\section{Before the improvement:}

For inner wall-1, Rw value $31(\mathbf{0} ;-\mathbf{1})$, DnT value $30(\mathbf{0} ; \mathbf{- 1})$

For inner wall-2, Rw value $40(-\mathbf{1} ;-\mathbf{3})$, DnT value $40(-\mathbf{1} ;-\mathbf{3})$

\section{After the improvement:}

For inner wall-1, Rw value $31(\mathbf{0} ; \mathbf{0})$, DnT value $30(\mathbf{0} ; \mathbf{- 1})$

For inner wall-2, Rw value $39(-\mathbf{1} ;-3)$, DnT value $39(-\mathbf{1} ;-\mathbf{3})$

These fall below the required limit values (Table 10). 
Table 9. Comparison of sound loss values before and after the application

\begin{tabular}{|c|c|c|c|c|c|c|}
\hline \multirow{2}{*}{$\begin{array}{c}\text { Frequency } \\
\qquad(\mathrm{Hz})\end{array}$} & \multicolumn{3}{|c|}{ Inner wall-1 } & \multicolumn{3}{|c|}{ Inner wall-2 } \\
\hline & $\begin{array}{c}\text { Before } \\
\text { Application (dB) }\end{array}$ & $\begin{array}{c}\text { After } \\
\text { Application (dB) }\end{array}$ & $\begin{array}{l}\text { Difference } \\
\text { (dB) }\end{array}$ & $\begin{array}{c}\text { Before Application } \\
\text { (dB) }\end{array}$ & $\begin{array}{l}\text { After Application } \\
\text { (dB) }\end{array}$ & $\begin{array}{l}\text { Difference } \\
\text { (dB) }\end{array}$ \\
\hline 100 & 36,0 & 32,2 & 3,8 & 31,6 & 25,9 & 5,7 \\
\hline 125 & 34,3 & 28,0 & 6,3 & 31,8 & 30,7 & 1,1 \\
\hline 160 & 31,7 & 28,2 & 3,5 & 31,1 & 29,1 & 2,0 \\
\hline 200 & 35,3 & 26,3 & 9,0 & 29,7 & 30,2 & $-0,5$ \\
\hline 250 & 36,2 & 33,5 & 2,7 & 30,4 & 29,2 & 1,2 \\
\hline 315 & 35,5 & 30,1 & 5,4 & 29,7 & 28,6 & 1,1 \\
\hline 400 & 37,0 & 29,0 & 8,0 & 34,4 & 33,4 & 1,0 \\
\hline 500 & 36,6 & 29,4 & 7,2 & 36,5 & 36,8 & $-0,3$ \\
\hline 630 & 38,0 & 32,4 & 5,6 & 40,3 & 39,6 & 0,7 \\
\hline 800 & 37,6 & 29,7 & 7,9 & 41,4 & 39,6 & 1,8 \\
\hline 1000 & 37,7 & 32,5 & 5,2 & 40,0 & 41,2 & $-1,2$ \\
\hline 1250 & 36,9 & 31,8 & 5,1 & 44,8 & 42,2 & 2,6 \\
\hline 1600 & 37,1 & 31,4 & 5,7 & 43,1 & 42,4 & 0,7 \\
\hline 2000 & 37,4 & 31,4 & 6,0 & 41,7 & 39,7 & 2,0 \\
\hline 2500 & 36,9 & 30,9 & 6,0 & 42,1 & 39,7 & 2,4 \\
\hline 3150 & 37,6 & 31,8 & 5,8 & 43,7 & 40,8 & 2,9 \\
\hline 4000 & 39,8 & 33,7 & 6,1 & 47,1 & 44,5 & 2,6 \\
\hline 5000 & 41,7 & 35,8 & 5,9 & 47,9 & 46,9 & 1,0 \\
\hline
\end{tabular}

For the inner wall-1, the pre- and post-improvement values decreased between 2.7 to $9.0 \mathrm{~dB}$. This suggests a decrease in the acoustic performance of the building element. Considering the differences in the inner wall-1 before and after the improvement, the sound transmission loss value appears to decrease more at high frequencies as compared to other frequencies. Therefore, it is possible to draw the conclusion that the silk (live) plaster used was not as effective at high frequencies. Furthermore, it is accepted that the sound transmission loss value was reduced because of the composite nature (door + wall) of the inner wall-1. In composite structures, values obtained are close to the sound transmission loss value of the component with the lower performance.

The evaluation performed before and after the improvement for the inner wall-2 showed that the sound transmission loss value of the building element decreased with a difference of $5.7 \mathrm{~dB}$ at maximum 100 $\mathrm{Hz}$ frequency and $2.9 \mathrm{~dB}$ at other frequencies. After the improvement application at frequencies of 200, 500 , and $1000 \mathrm{~Hz}$, the performance seemed to increase albeit at minimal levels.

It was observed that the application of silk (live) plaster on the building element did not have any positive effect on the sound transmission loss value. It was not possible to obtain the $\mathrm{R}_{\mathrm{w}}$ values of silk (living) plaster material on the basis of frequency (as required) through a review of literature and the material catalogs for simulation programs or by reaching out to companies that market and implement the material. This study highlights the need to perform laboratory analyses of silk (live) plaster material on the basis of frequency.

Mass value is very important in sound-related applications. And it should be noted that the said material has no mass value. Considering the volume acoustics properties of the material, the decrease in the reverberation time at high frequencies was expected (Figure 7). In the specific example of the educational facilities, it is possible to talk about an improvement, even though it did not meet the required limit values. 


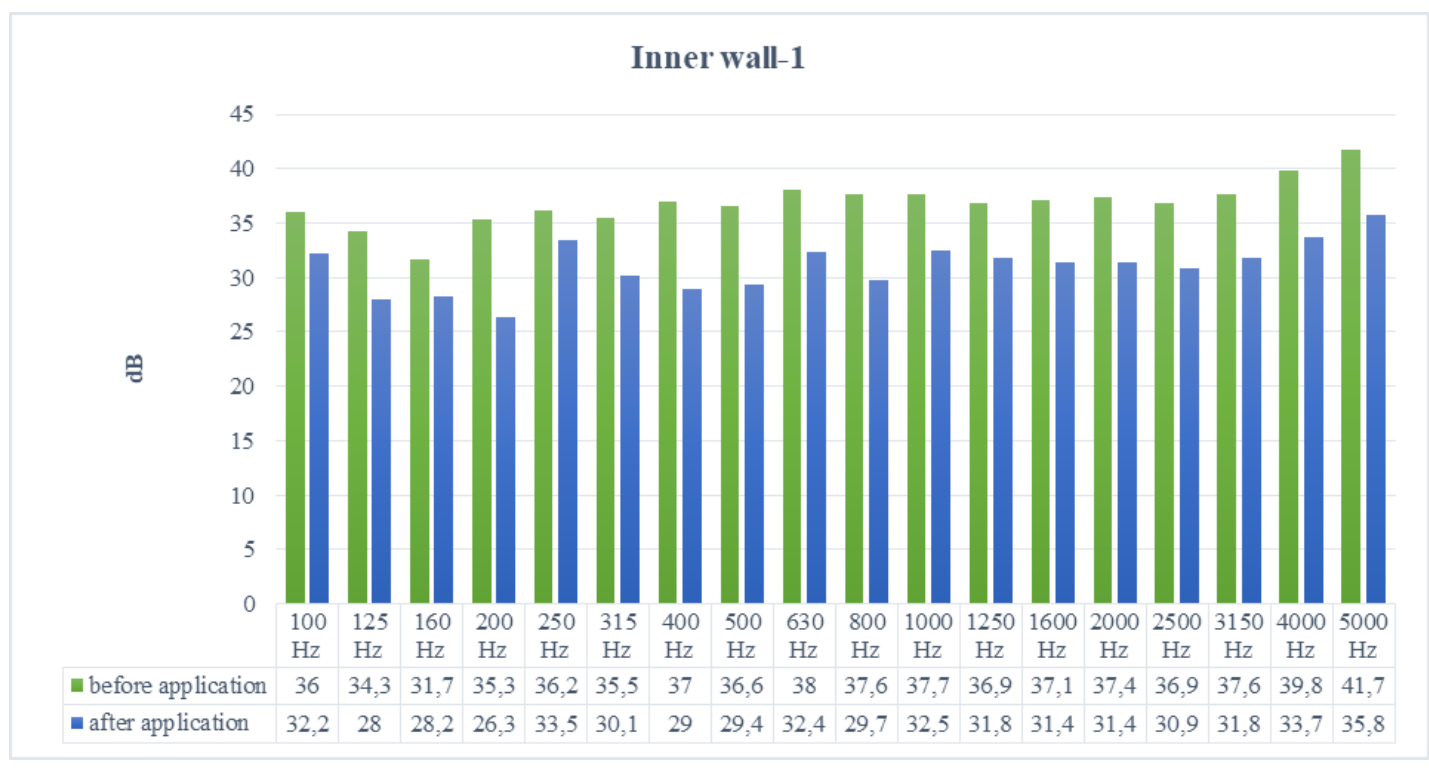

Figure 8. Sound transition loss values before and after the application

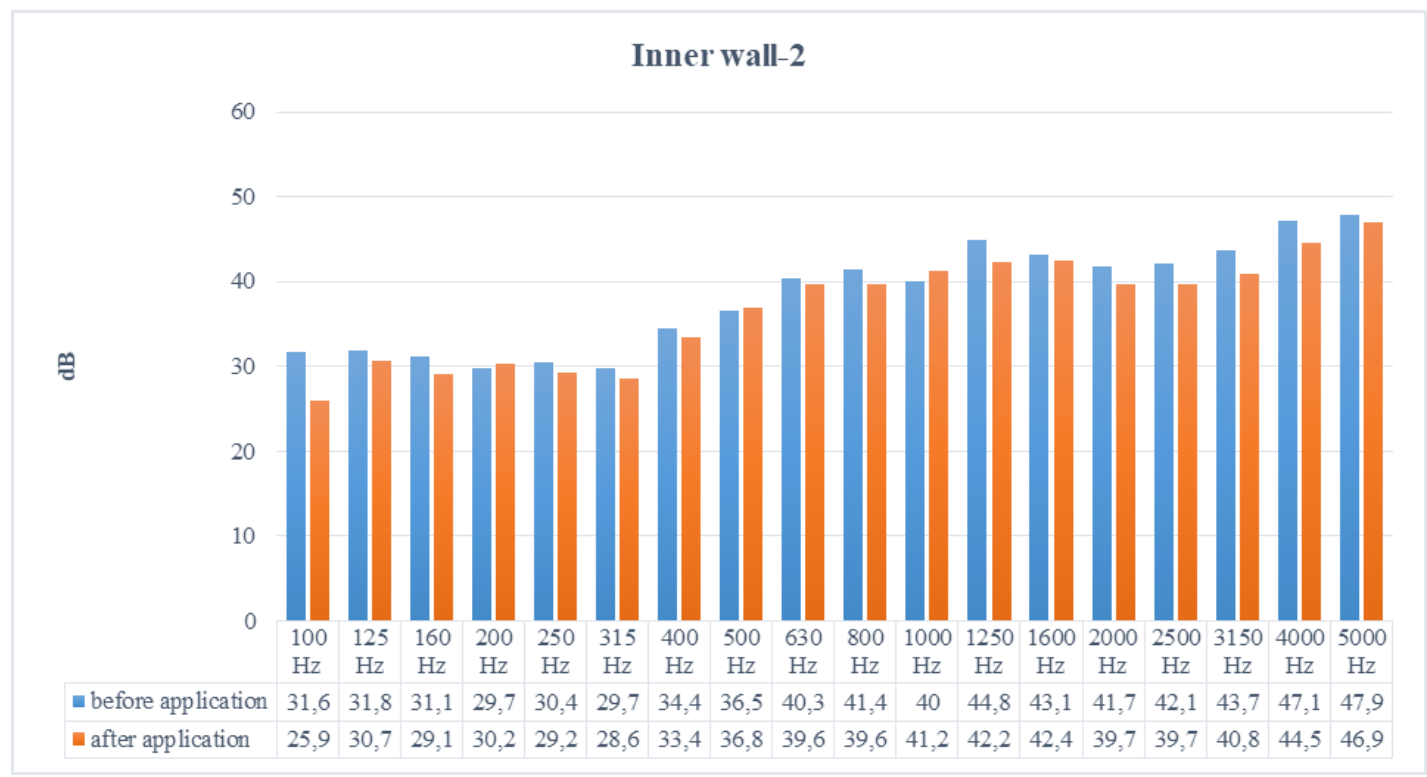

Figure 9. Pre- and post-improvement sound transmission loss values

Table 10. Assessment results

\begin{tabular}{|c|c|c|c|c|}
\hline \multirow[b]{2}{*}{$\begin{array}{l}\text { Building } \\
\text { Element }\end{array}$} & \multirow{2}{*}{$\begin{array}{l}\text { Optimum Value "Regulation } \\
\text { on Protection of Buildings } \\
\text { Against Noise" }\end{array}$} & \multicolumn{2}{|c|}{ Measurement and Calculation Results } & \multirow[b]{2}{*}{ Evaluation } \\
\hline & & Before Application & After Application & \\
\hline $\begin{array}{l}\text { Inner Wall - 1 } \\
\text { (Compound } \\
\text { wall) }\end{array}$ & $\begin{array}{c}\mathrm{D}_{\mathrm{nT,A}}=48 \mathrm{~dB} \\
\text { Due to the door } \\
48-14=34 \mathrm{~dB} \text { (for class } \mathrm{D})\end{array}$ & $\begin{array}{c}\mathrm{R}_{\mathrm{w}}(\mathrm{C} ; \mathrm{Ctr}) \\
31(0 ;-1) \\
\mathrm{D}_{\mathrm{nT}, \mathrm{w}} 30(0 ;-1)\end{array}$ & $\begin{array}{c}\mathrm{R}_{\mathrm{w}}(\mathrm{C} ; \mathrm{Ctr}) \\
31(0 ; 0) \\
\mathrm{D}_{\mathrm{nT}, \mathrm{w}} 30(0 ;-1)\end{array}$ & $\begin{array}{c}\text { NOT } \\
\text { AVAILABLE }\end{array}$ \\
\hline Inner Wall - 2 & $\mathrm{D}_{\mathrm{nT}, \mathrm{A}}=48 \mathrm{~dB}$ (for class $\mathrm{D}$ ) & $\begin{array}{c}\mathrm{R}_{\mathrm{w}}(\mathrm{C} ; \mathrm{Ctr}) \\
40(-1 ;-3) \\
\mathrm{D}_{\mathrm{nT}, \mathrm{w}} 40(-1 ;-3)\end{array}$ & $\begin{array}{c}\mathrm{R}_{\mathrm{w}}(\mathrm{C} ; \mathrm{Ctr}) \\
39(-1 ;-3) \\
\mathrm{D}_{\mathrm{nT}, \mathrm{w}} 39(-1 ;-3)\end{array}$ & $\begin{array}{c}\text { NOT } \\
\text { AVAILABLE }\end{array}$ \\
\hline \multicolumn{5}{|c|}{$\begin{array}{l}\mathrm{D}_{\mathrm{nT}} \text { values } \mathrm{D}_{\mathrm{nT,w}}=\mathrm{R}^{\prime}{ }_{\mathrm{w}}+10 \log \left(\frac{0.16 \mathrm{~V}}{T_{0} S_{s}}\right)=\mathrm{R}_{\mathrm{w}}{ }_{\mathrm{w}}+10 \log \left(\frac{0.32 \mathrm{~V}}{S_{s}}\right) \text { It is calculated according to the formula. } \\
\mathrm{D}_{\mathrm{nT,A}}=\mathrm{D}_{\mathrm{nT, \textrm {w }}}+\mathrm{C}\end{array}$} \\
\hline
\end{tabular}


Table 10 shows the evaluation in light of the limit values as required by the "Regulation on the Protection of Buildings against Noise." According to this table, the class D required value in education buildings is $\mathrm{D}_{\mathrm{nT}, \mathrm{A}}=48 \mathrm{~dB}$. With a door added, $14 \mathrm{~dB}$ can be considered low. $\mathrm{D}_{\mathrm{nT}, \mathrm{A}}$ equals $34 \mathrm{~dB}$.

For the inner walls -1 and -2 , it was determined that the pre- and post-improvement sound transmission loss values were below the limits (Table 10). Silk (live) plaster is known as a building material that provides acoustic effects, besides heat and sound insulation. It did not provide the desired limit values in this implementation, but better results are likely with increased material thickness. It is recommended to examine the building material by recording its performance at each frequency, in order to improve acoustic comfort and, in particular, the performance of the building elements. Furthermore, in educational facilities with high sensitivity, a better outcome can be expected of improvement efforts with the use of materials tested for sound insulation and designed on location.

This study provided a comparison of the interior surface finishing material and the performance of building elements using the on-site measurement method. However, the method of on-site measurement may not be suitable for all circumstances. In fact, the significance and contribution of this study are based on the use of a well-known material in the measurements performed before and after the implementation of improvements.

\section{References}

[1] Yılmaz Demirkale, S., (2007). Çevre ve yapı akustiği, İstanbul, Türkiye: Birsen Yayınevi, ss. 202-465.

[2] Kurra, S., (1997). Çevre gürültüsü kirliliği ve kontrolü, Gürültü ile Savaşım Sempozyumu, İstanbul, Türkiye: ss. 31-40.

[3] Kurra, S., (2009). Çevre gürültüsü ve yönetimi, I-II-III, İstanbul, Türkiye: Bahçeşehir Üniversitesi Yayınları, ss. 55-460.

[4] Bayazıt, N. T., (2006). Sürdürülebilirlik Açısından Akustik Tasarım: İlköğretim Binaları Örneği, Tasarım Dergisi, sayı: 157, 130-133.

[5] Gökçora, B., (2003). Okullarda Akustik ve Konuşmanın Anlaşılabilirliği, İzolasyon Dünyası, 54.

[6] Can, Z, Y., (2007). Eğitim Yapılarında Gürültü ve Denetimi, İzolasyon Dünyası Dergisi, sayı: 66, 16.

[7] Öztürk, İ., (2001). The Role Of Education In Economic Development: A Theoretical Perspective, Journal of Rural Development and Administration, Vol. XXXIII, No. 1, pp. 3947.

[8] Esin, T., (2007). Eğitim Yapılarında Konfor Koşullarının Sağlanması ve Yalıtım, İzolasyon Dünyas1, Sayı 66, ss.71-74.

[9] Petreche, Diego, R., (2007). An evaluation method for school building design at the preliminary phase with optimisation of aspects of environmental comfort for the school system of the State Sa o Paulo in Brazil, Building and Environment, Vol. 42, Issue 2, pp. 984999.

[10] Sutherland, L.C. and Lubman, D., (2001). The Impact of Classroom Acoustics on Scholastic Achievement, 17th Meeting of the International Commission for Acoustics, Rome, Italy, Sept. ss. 2-7.

[11] Federal Interagency Committee On Aviation Noise (Fican) (2000). FICAN Position on Research into Effects of Aircraft Noise on Classroom Learning, Washington, D.C. 
[12] TS EN ISO 16283-1, (2014). Akustik-Yapı Elemanlarında ve Yapılarda Ses Yalıtımının Alan Ölçümü-Bölüm 1: Hava ile Yayılan Sesin Yalıtımı.

[13] TS EN ISO 717-1, (2013). Akustik-Yapılarda ve Yapı Elemanlarında Ses Yalıtımının Değerlendirilmesi-Bölüm 1: Hava ile Yayılan Sesin Yalıtımı.

[14] Binaların Gürültüye Karşı Korunması Hakkındaki Yönetmelik, (2017). Resmi Gazete, Sayı: 30082 . 
International Journal of Scientific and Technological Research

ISSN 2422-8702 (Online), DOI: 10.7176/JSTR/6-04-11

Vol.6, No.4, 2020

\section{ANNEX}

Table 1. For Inner Wall- 1; C, Ctr data (One-third octave) (Before Application)

\begin{tabular}{|c|c|c|c|c|c|c|c|c|c|}
\hline Frequency & $\mathbf{R}_{\mathbf{w}}$ & $\begin{array}{l}\text { Reference } \\
\text { values } \\
\text { shifted by } \\
-21 \text { dB }\end{array}$ & $\begin{array}{c}\text { Unfavourable } \\
\text { deviation } \\
\text { dB }\end{array}$ & $\begin{array}{c}\text { Spectrum } \\
\text { No:1 }\left(L_{n}\right) \\
\text { dB }\end{array}$ & $\begin{array}{c}L_{n}-R_{w} \\
d B\end{array}$ & $\begin{array}{c}10^{(\operatorname{Ln}-\mathrm{R} w) / 10} \\
\mathrm{dBx} 10^{-5}\end{array}$ & $\begin{array}{c}\text { Spectrum } \\
\text { No:2 }\left(L_{n}\right) \\
\text { dB }\end{array}$ & $\mathbf{L}_{\mathbf{n}}-\mathbf{R}_{\mathbf{w}}$ & $10^{(\mathrm{Ln}-\mathrm{R} w) / 10}$ \\
\hline 100 & 29,6 & 12 & - & -29 & $-58,6$ & 0,138038426 & -20 & $-49,6$ & 1,096478196 \\
\hline 125 & 27,9 & 15 & - & -26 & $-53,9$ & 0,407380278 & -20 & $-47,9$ & 1,621810097 \\
\hline 160 & 25,3 & 18 & - & -23 & $-48,3$ & 1,479108388 & -18 & $-43,3$ & 4,677351413 \\
\hline 200 & 28,9 & 21 & - & -21 & $-49,9$ & 1,023292992 & -16 & $-44,9$ & 3,235936569 \\
\hline 250 & 29,8 & 24 & - & -19 & $-48,8$ & 1,318256739 & -15 & $-44,8$ & 3,311311215 \\
\hline 315 & 29,1 & 27 & - & -17 & $-46,1$ & 2,454708916 & -14 & $-43,1$ & 4,897788194 \\
\hline 400 & 30,6 & 30 & - & -15 & $-45,6$ & 2,754228703 & -13 & $-43,6$ & 4,365158322 \\
\hline 500 & 30,2 & 31 & 0,8 & -13 & $-43,2$ & 4,786300923 & -12 & $-42,2$ & 6,025595861 \\
\hline 630 & 31,6 & 32 & 0,4 & -12 & $-43,6$ & 4,365158322 & -11 & $-42,6$ & 5,495408739 \\
\hline 800 & 31,2 & 33 & 1,8 & -11 & $-42,2$ & 6,025595861 & -9 & $-40,2$ & 9,54992586 \\
\hline 1000 & 31,3 & 34 & 2,7 & -10 & $-41,3$ & 7,413102413 & -8 & $-39,3$ & 11,74897555 \\
\hline 1250 & 30,5 & 35 & 4,5 & -9 & $-39,5$ & 11,22018454 & -9 & $-39,5$ & 11,22018454 \\
\hline 1600 & 30,6 & 35 & 4,4 & -9 & $-39,6$ & 10,96478196 & -10 & $-40,6$ & 8,7096359 \\
\hline 2000 & 30,9 & 35 & 4,1 & -9 & $-39,9$ & 10,23292992 & -11 & $-41,9$ & 6,45654229 \\
\hline 2500 & 30,5 & 35 & 4,5 & -9 & $-39,5$ & 11,22018454 & -13 & $-43,5$ & 4,466835922 \\
\hline \multirow[t]{2}{*}{3150} & 31,2 & 35 & 3,8 & -9 & $-40,2$ & 9,54992586 & -15 & $-46,2$ & 2,398832919 \\
\hline & \multicolumn{3}{|c|}{$\begin{array}{c}\operatorname{sum}=27<32,0 \\
=52-21 \mathrm{~dB}=31 \mathrm{~dB}\end{array}$} & \multicolumn{3}{|c|}{$\mathrm{C}=0$} & \multicolumn{3}{|c|}{$\mathrm{C}_{\mathrm{tr}}=-1$} \\
\hline
\end{tabular}

Table 2. For Inner Wall- 2; C, Ctr data (One-third octave) (Before Application)

\begin{tabular}{|c|c|c|c|c|c|c|c|c|c|}
\hline Frequency & $\mathbf{R}_{\mathbf{w}}$ & $\begin{array}{l}\text { Reference } \\
\text { values } \\
\text { shifted by } \\
-12 \mathrm{~dB}\end{array}$ & $\begin{array}{c}\text { Unfavourable } \\
\text { deviation } \\
\text { dB }\end{array}$ & $\begin{array}{c}\text { Spectrum } \\
\operatorname{No:1}\left(L_{n}\right) \\
\text { dB }\end{array}$ & $\begin{array}{c}L_{n}-R_{w} \\
d B\end{array}$ & $\begin{array}{l}10^{(\operatorname{Ln}-\mathrm{R} w) / 10} \\
\mathrm{dBx}^{-5} 0^{-5}\end{array}$ & $\begin{array}{c}\text { Spectrum } \\
\text { No:2 }\left(L_{n}\right) \\
\text { dB }\end{array}$ & $\begin{array}{c}\mathbf{L}_{\mathbf{n}}-\mathbf{R}_{\mathrm{w}} \\
\mathrm{dB}\end{array}$ & $\begin{array}{c}10^{(\mathrm{Ln}-\mathrm{Rw}) / 10} \\
\mathrm{dB \times 10^{-5 }}\end{array}$ \\
\hline 100 & 31,6 & 21 & --- & -29 & $-60,6$ & 0,087096359 & -20 & $-51,6$ & 0,691830971 \\
\hline 125 & 31,8 & 24 & --- & -26 & $-57,8$ & 0,165958691 & -20 & $-51,8$ & 0,660693448 \\
\hline 160 & 31,1 & 27 & --- & -23 & $-54,1$ & 0,389045145 & -18 & $-49,1$ & 1,230268771 \\
\hline 200 & 29,7 & 30 & 0,3 & -21 & $-50,7$ & 0,851138038 & -16 & $-45,7$ & 2,691534804 \\
\hline 250 & 30,4 & 33 & 2,6 & -19 & $-49,4$ & 1,148153621 & -15 & $-45,4$ & 2,884031503 \\
\hline 315 & 29,7 & 36 & 6,3 & -17 & $-46,7$ & 2,13796209 & -14 & $-43,7$ & 4,265795188 \\
\hline 400 & 34,4 & 39 & 4,6 & -15 & $-49,4$ & 1,148153621 & -13 & $-47,4$ & 1,819700859 \\
\hline 500 & 36,5 & 40 & 3,5 & -13 & $-49,5$ & 1,122018454 & -12 & $-48,5$ & 1,412537545 \\
\hline 630 & 40,3 & 41 & 0,7 & -12 & $-52,3$ & 0,588843655 & -11 & $-51,3$ & 0,741310241 \\
\hline 800 & 41,4 & 42 & 0,6 & -11 & $-52,4$ & 0,575439937 & -9 & $-50,4$ & 0,912010839 \\
\hline 1000 & 40 & 43 & 3 & -10 & -50 & 1 & -8 & -48 & 1,584893192 \\
\hline 1250 & 44,8 & 44 & --- & -9 & $-53,8$ & 0,416869383 & -9 & $-53,8$ & 0,416869383 \\
\hline 1600 & 43,1 & 44 & 0,9 & -9 & $-52,1$ & 0,616595002 & -10 & $-53,1$ & 0,489778819 \\
\hline 2000 & 41,7 & 44 & 2,3 & -9 & $-50,7$ & 0,851138038 & -11 & $-52,7$ & 0,537031796 \\
\hline 2500 & 42,1 & 44 & 1,9 & -9 & $-51,1$ & 0,776247117 & -13 & $-55,1$ & 0,309029543 \\
\hline 3150 & 43,7 & 44 & 0,3 & -9 & $-52,7$ & 0,537031796 & -15 & $-58,7$ & 0,134896288 \\
\hline & \multicolumn{3}{|c|}{$\begin{array}{c}\operatorname{sum}=27<32,0 \\
\mathrm{R}_{\mathrm{w}}=52-12 \mathrm{~dB}=40 \mathrm{~dB}\end{array}$} & \multicolumn{3}{|c|}{$\mathrm{C}=-1$} & \multicolumn{3}{|c|}{$\mathrm{C}_{\mathrm{tr}}=-3$} \\
\hline
\end{tabular}


International Journal of Scientific and Technological Research

ISSN 2422-8702 (Online), DOI: 10.7176/JSTR/6-04-11

Vol.6, No.4, 2020

Table 3. For Inner Wall- 1; C, Ctr data (One-third octave) (After Application)

\begin{tabular}{|c|c|c|c|c|c|c|c|c|c|}
\hline Frequency & $\mathbf{R}_{\mathrm{w}}$ & $\begin{array}{l}\text { Reference } \\
\text { values } \\
\text { shifted by } \\
-21 \text { dB }\end{array}$ & $\begin{array}{c}\text { Unfavourable } \\
\text { deviation } \\
\text { dB }\end{array}$ & $\begin{array}{c}\text { Spectrum } \\
\text { No:1 }\left(L_{n}\right) \\
\text { dB }\end{array}$ & $\begin{array}{c}\mathbf{L}_{\mathbf{n}}-\mathbf{R}_{\mathrm{w}} \\
\mathrm{dB}\end{array}$ & $\begin{array}{l}10^{(\operatorname{Ln}-\mathrm{Rw}) / 10} \\
\mathrm{~dB}^{-10} 10^{-5}\end{array}$ & $\begin{array}{c}\text { Spectrum } \\
\text { No:2 }\left(L_{n}\right) \\
\text { dB }\end{array}$ & $\begin{array}{c}\mathbf{L}_{\mathrm{n}}-\mathbf{R}_{\mathrm{w}} \\
\mathrm{dB}\end{array}$ & $\begin{array}{c}10^{(\operatorname{Ln}-\mathrm{Rw}) / 10} \\
\mathrm{~dB} \times 10^{-5}\end{array}$ \\
\hline 100 & 32,2 & 12 & --- & -29 & $-61,2$ & 0,075857758 & -20 & $-52,2$ & 0,602559586 \\
\hline 125 & 28 & 15 & --- & -26 & -54 & 0,398107171 & -20 & -48 & 1,584893192 \\
\hline 160 & 28,2 & 18 & --- & -23 & $-51,2$ & 0,758577575 & -18 & $-46,2$ & 2,398832919 \\
\hline 200 & 26,3 & 21 & --- & -21 & $-47,3$ & 1,862087137 & -16 & $-42,3$ & 5,888436554 \\
\hline 250 & 33,5 & 24 & --- & -19 & $-52,5$ & 0,562341325 & -15 & $-48,5$ & 1,412537545 \\
\hline 315 & 30,1 & 27 & --- & -17 & $-47,1$ & 1,9498446 & -14 & $-44,1$ & 3,89045145 \\
\hline 400 & 29 & 30 & 1 & -15 & -44 & 3,981071706 & -13 & -42 & 6,309573445 \\
\hline 500 & 29,4 & 31 & 1,6 & -13 & $-42,4$ & 5,754399373 & -12 & $-41,4$ & 7,244359601 \\
\hline 630 & 32,4 & 32 & --- & -12 & $-44,4$ & 3,630780548 & -11 & $-43,4$ & 4,570881896 \\
\hline 800 & 29,7 & 33 & 3,3 & -11 & $-40,7$ & 8,511380382 & -9 & $-38,7$ & 13,48962883 \\
\hline 1000 & 32,5 & 34 & 1,5 & -10 & $-42,5$ & 5,623413252 & -8 & $-40,5$ & 8,912509381 \\
\hline 1250 & 31,8 & 35 & 3,2 & -9 & $-40,8$ & 8,317637711 & -9 & $-40,8$ & 8,317637711 \\
\hline 1600 & 31,4 & 35 & 3,6 & -9 & $-40,4$ & 9,120108394 & -10 & $-41,4$ & 7,244359601 \\
\hline 2000 & 31,4 & 35 & 3,6 & -9 & $-40,4$ & 9,120108394 & -11 & $-42,4$ & 5,754399373 \\
\hline 2500 & 30,9 & 35 & 4,1 & -9 & $-39,9$ & 10,23292992 & -13 & $-43,9$ & 4,073802778 \\
\hline 3150 & 31,8 & 35 & & -9 & $-40,8$ & 8,317637711 & -15 & $-46,8$ & 2,089296131 \\
\hline & \multicolumn{3}{|c|}{$\begin{array}{c}\operatorname{sum}=21,9<32,0 \\
\mathrm{R}_{\mathrm{w}}=52-21 \mathrm{~dB}=31 \mathrm{~dB}\end{array}$} & \multicolumn{3}{|c|}{$\mathrm{C}=0$} & \multicolumn{3}{|c|}{$\mathrm{C}_{\mathrm{tr}}=0$} \\
\hline
\end{tabular}

Table 4. For Inner Wall- 2; C, Ctr data (One-third octave) (After Application)

\begin{tabular}{|c|c|c|c|c|c|c|c|c|c|}
\hline Frequency & $\mathbf{R}_{\mathrm{w}}$ & $\begin{array}{c}\text { Reference } \\
\text { values shifted } \\
\text { by } \mathbf{- 1 3} \text { dB dB }\end{array}$ & $\begin{array}{c}\text { Unfavourable } \\
\text { deviation } \\
\text { dB }\end{array}$ & $\begin{array}{c}\text { Spectrum } \\
\text { No:1 }\left(L_{n}\right) \\
\text { dB }\end{array}$ & $\begin{array}{c}\mathbf{L}_{\mathbf{n}}-\mathbf{R}_{\mathrm{w}} \\
\mathrm{dB}\end{array}$ & $\begin{array}{l}10^{(\operatorname{Ln}-R w) / 10} \\
\text { dBx10 }^{-5}\end{array}$ & $\begin{array}{c}\text { Spectrum } \\
\text { No:2 }\left(L_{n}\right) \\
\text { dB }\end{array}$ & $\begin{array}{c}L_{\mathbf{n}}-\mathbf{R}_{\mathbf{w}} \\
\text { dB }\end{array}$ & $\begin{array}{c}10^{(\operatorname{Ln}-\mathrm{R} w) / 10} \\
\mathrm{~dB} \times 10^{-5}\end{array}$ \\
\hline 100 & 25,9 & 20 & --- & -29 & $-54,9$ & 0,3235937 & -20 & $-45,9$ & 2,5703958 \\
\hline 125 & 30,7 & 23 & --- & -26 & $-56,7$ & 0,2137962 & -20 & $-50,7$ & 0,851138 \\
\hline 160 & 29,1 & 26 & --- & -23 & $-52,1$ & 0,616595 & -18 & $-47,1$ & 1,9498446 \\
\hline 200 & 30,2 & 29 & --- & -21 & $-51,2$ & 0,7585776 & -16 & $-46,2$ & 2,3988329 \\
\hline 250 & 29,2 & 32 & 2,8 & -19 & $-48,2$ & 1,5135612 & -15 & $-44,2$ & 3,801894 \\
\hline 315 & 28,6 & 35 & 6,4 & -17 & $-45,6$ & 2,7542287 & -14 & $-42,6$ & 5,4954087 \\
\hline 400 & 33,4 & 38 & 4,6 & -15 & $-48,4$ & 1,4454398 & -13 & $-46,4$ & 2,2908677 \\
\hline 500 & 36,8 & 39 & 2,2 & -13 & $-49,8$ & 1,0471285 & -12 & $-48,8$ & 1,3182567 \\
\hline 630 & 39,6 & 40 & 0,4 & -12 & $-51,6$ & 0,691831 & -11 & $-50,6$ & 0,8709636 \\
\hline 800 & 39,6 & 41 & 1,4 & -11 & $-50,6$ & 0,8709636 & -9 & $-48,6$ & 1,3803843 \\
\hline 1000 & 41,2 & 42 & 0,8 & -10 & $-51,2$ & 0,7585776 & -8 & $-49,2$ & 1,2022644 \\
\hline 1250 & 42,2 & 43 & 0,8 & -9 & $-51,2$ & 0,7585776 & -9 & $-51,2$ & 0,7585776 \\
\hline 1600 & 42,4 & 43 & 0,6 & -9 & $-51,4$ & 0,724436 & -10 & $-52,4$ & 0,5754399 \\
\hline 2000 & 39,7 & 43 & 3,3 & -9 & $-48,7$ & 1,3489629 & -11 & $-50,7$ & 0,851138 \\
\hline 2500 & 39,7 & 43 & 3,3 & -9 & $-48,7$ & 1,3489629 & -13 & $-52,7$ & 0,5370318 \\
\hline 3150 & 40,8 & 43 & 2,2 & -9 & $-49,8$ & 1,0471285 & -15 & $-55,8$ & 0,2630268 \\
\hline & \multicolumn{3}{|c|}{$\begin{array}{c}\text { sum }=28,8<32,0 \\
\mathrm{R}_{\mathrm{w}}=52-13 \mathrm{~dB}=39 \mathrm{~dB}\end{array}$} & \multicolumn{3}{|c|}{$C=-1$} & \multicolumn{3}{|c|}{$\mathrm{C}_{\mathrm{tr}}=-3$} \\
\hline
\end{tabular}

